\title{
The Star Formation in Radio Survey: Jansky Very Large Array 33 GHz Observations of Nearby Galaxy Nuclei and Extranuclear Star-forming Regions
}

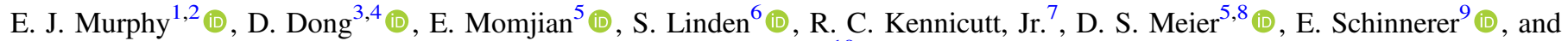 \\ J. L. Turner ${ }^{10}$ (i) \\ ${ }^{1}$ National Radio Astronomy Observatory, 520 Edgemont Road, Charlottesville, VA 22903, USA; emurphy@nrao.edu \\ ${ }^{2}$ Infrared Processing and Analysis Center, California Institute of Technology, MC 220-6, Pasadena, CA 91125, USA \\ ${ }^{3}$ Department of Physics and Astronomy, Pomona College, Claremont, CA 91711, USA \\ ${ }^{4}$ California Institute of Technology, MC 100-22, Pasadena, CA 91125, USA \\ ${ }^{5}$ National Radio Astronomy Observatory, P.O. Box O, 1003 Lopezville Road, Socorro, NM 87801, USA \\ ${ }^{6}$ Department of Astronomy, University of Virginia, 3530 McCormick Road, Charlottesville, VA 22904, USA \\ ${ }^{7}$ Institute of Astronomy, University of Cambridge, Madingley Road, Cambridge CB3 0HA, UK \\ ${ }^{8}$ New Mexico Institute of Mining and Technology, 801 Leroy Place, Socorro, NM 87801, USA \\ ${ }^{9}$ Max Planck Institut für Astronomie, Königstuhl 17, Heidelberg D-69117, Germany \\ ${ }^{10}$ Department of Physics and Astronomy, UCLA, Los Angeles, CA 90095, USA \\ Received 2017 August 3; revised 2017 November 7; accepted 2017 November 8; published 2018 February 1
}

\begin{abstract}
We present $33 \mathrm{GHz}$ imaging for 112 pointings toward galaxy nuclei and extranuclear star-forming regions at $\approx 2^{\prime \prime}$ resolution using the Karl G. Jansky Very Large Array (VLA) as part of the Star Formation in Radio Survey. A comparison with $33 \mathrm{GHz}$ Robert C. Byrd Green Bank Telescope single-dish observations indicates that the interferometric VLA observations recover $78 \% \pm 4 \%$ of the total flux density over $25^{\prime \prime}$ regions ( $\approx \mathrm{kpc}$ scales) among all fields. On these scales, the emission being resolved out is most likely diffuse non-thermal synchrotron emission. Consequently, on the $\approx 30-300 \mathrm{pc}$ scales sampled by our VLA observations, the bulk of the $33 \mathrm{GHz}$ emission is recovered and primarily powered by free-free emission from discrete $\mathrm{H}$ II regions, making it an excellent tracer of massive star formation. Of the 225 discrete regions used for aperture photometry, 162 are extranuclear (i.e., having galactocentric radii $r_{\mathrm{G}} \geqslant 250 \mathrm{pc}$ ) and detected at $>3 \sigma$ significance at $33 \mathrm{GHz}$ and in $\mathrm{H} \alpha$. Assuming a typical $33 \mathrm{GHz}$ thermal fraction of $90 \%$, the ratio of optically-thin $33 \mathrm{GHz}$ to uncorrected $\mathrm{H} \alpha$ star formation rates indicates a median extinction value on $\approx 30-300$ pc scales of $A_{\mathrm{H} \alpha} \approx 1.26 \pm 0.09 \mathrm{mag}$, with an associated median absolute deviation of $0.87 \mathrm{mag}$. We find that $10 \%$ of these sources are "highly embedded" (i.e., $A_{\mathrm{H} \alpha} \gtrsim 3.3 \mathrm{mag}$ ), suggesting that on average, $\mathrm{H}$ II regions remain embedded for $\lesssim 1$ Myr. Finally, we find the median $33 \mathrm{GHz}$ continuum-to-H $\alpha$ line flux ratio to be statistically larger within $r_{\mathrm{G}}<250 \mathrm{pc}$ relative to the outer disk regions by a factor of $1.82 \pm 0.39$, while the ratio of $33 \mathrm{GHz}$ to $24 \mu \mathrm{m}$ flux densities is lower by a factor of $0.45 \pm 0.08$, which may suggest increased extinction in the central regions.
\end{abstract}

Key words: galaxies: nuclei - H II regions - radio continuum: general - stars: formation

Supporting material: extended figure, machine-readable tables

\section{Introduction}

Radio emission from galaxies is powered by a combination of distinct physical processes. And although it is energetically weak with respect to a galaxy's bolometric luminosity, it provides critical information on the massive star formation activity, as well as access to the relativistic [magnetic field + cosmic rays (CRs)] component in the interstellar medium (ISM) of galaxies.

Stars more massive than $\sim 8 M_{\odot}$ end their lives as corecollapse supernovae, whose remnants are thought to be the primary accelerators of $\mathrm{CR}$ electrons (e.g., Koyama et al. 1995), giving rise to the diffuse synchrotron emission observed from star-forming galaxies (Condon 1992). These same massive stars are also responsible for the creation of $\mathrm{H}$ II regions that produce radio free-free emission, whose strength is directly proportional to the production rate of ionizing (Lyman continuum) photons.

Radio frequencies spanning $\sim 1-100 \mathrm{GHz}$, which are observable from the ground, are particularly useful for probing such processes. The non-thermal emission component typically has a steep spectrum $\left(S_{\nu} \propto \nu^{-\alpha}\right.$, where $\left.\alpha \sim 0.8\right)$, while the thermal (free-free) component is relatively flat $(\alpha \sim 0.1$; e.g.,
Condon 1992). Accordingly, for globally integrated measurements of star-forming galaxies, lower frequencies (e.g., $1.4 \mathrm{GHz}$ ) are generally dominated by non-thermal emission, while the observed thermal fraction of the emission increases with frequency, eventually being dominated by free-free emission once beyond $\sim 30 \mathrm{GHz}$ (Condon \& Yin 1990). For typical $\mathrm{H}$ II regions, the thermal fraction at $33 \mathrm{GHz}$ can be considerably higher, being $\sim 80 \%$ (Murphy et al. 2011). Thus, observations at such frequencies, which are largely unbiased by dust, provide an excellent diagnostic for the current star formation rate (SFR) of galaxies.

It is worth noting that the presence of an anomalous microwave emission (AME) component in excess of free-free emission between $\sim 10$ and $90 \mathrm{GHz}$, generally attributed to electric dipole rotational emission from ultrasmall $\left(a \lesssim 10^{-6} \mathrm{~cm}\right)$ grains (e.g., Erickson 1957; Draine \& Lazarian 1998a, 1998b; Planck Collaboration et al. 2011) or magnetic dipole emission from thermal fluctuations in the magnetization of interstellar dust grains (Draine \& Lazarian 1999; Hensley et al. 2016), may complicate this picture. For a single outer disk star-forming region in NGC 6946, Murphy et al. (2010) reported an excess of $33 \mathrm{GHz}$ emission relative to what is expected given existing lowerfrequency radio data. This result has been interpreted as the first 
detection of so-called "anomalous" dust emission outside of the Milky Way. While the excess was only detected for a single region in this initial study, follow-up observations yielded additional detections in the disk of NGC 6946 (Hensley et al. 2015). However, it appears that this emission component is most likely sub-dominant for globally integrated measurements.

Due to the faintness of galaxies at high (i.e., $\gtrsim 15 \mathrm{GHz}$ ) radio frequencies, existing work has been restricted to the brightest objects, and small sample sizes. For example, past studies demonstrating the link between high-frequency free-free emission and massive star formation include investigations of Galactic star-forming regions (e.g., Mezger \& Henderson 1967), nearby dwarf irregular galaxies (e.g., Klein \& Graeve 1986), galaxy nuclei (e.g., Turner \& Ho 1983, 1994), nearby starbursts (e.g., Turner \& Ho 1985; Klein et al. 1988), and super star clusters within nearby blue compact dwarfs (e.g., Turner et al. 1998; Kobulnicky \& Johnson 1999). And while these studies focus on the free-free emission from galaxies, each was conducted at frequencies $\lesssim 30 \mathrm{GHz}$. With recent improvements to the backends of existing radio telescopes, such as the Caltech Continuum Backend (CCB) on the Robert C. Byrd Green Bank Telescope (GBT) and the Wideband Interferometric Digital ARchitecture (WIDAR) correlator on the Karl G. Jansky Very Large Array (VLA), the availability of increased bandwidth is making it possible to conduct investigations for large samples of objects at frequencies $\sim 30 \mathrm{GHz}$.

In a recent paper, we presented $33 \mathrm{GHz}$ photometry taken with the CCB on the GBT as part of the Star Formation in Radio Survey (SFRS; Murphy et al. 2012). Building on that work, we obtained $33 \mathrm{GHz}$ imaging for the SFRS using the VLA, allowing us to map the $33 \mathrm{GHz}$ emission from each region on $\gtrsim 2^{\prime \prime}$ scales, compared to the $\approx 25^{\prime \prime}$ single-beam GBT photometry. These galaxies, which are included in the Spitzer Infrared Nearby Galaxies Survey (SINGS; Kennicutt et al. 2003) and Key Insights on Nearby Galaxies: a FarInfrared Survey with Herschel (KINGFISH; Kennicutt et al. 2011) legacy programs, are well studied and have a wealth of ancillary data available. We are currently in the process of reducing and imaging complementary interferometric observations at matched resolution in the S- (2-4 GHz) and $\mathrm{Ku}-(12-18 \mathrm{GHz}$ ) bands (VLA/13B-215; PI. Murphy), which will allow us to extend this analysis by making spectral index maps and doing proper thermal/non-thermal decompositions. The complete multi-band survey data and associated full analysis will be presented in a forthcoming paper.

In this paper, we present catalogs of $33 \mathrm{GHz}$ images and flux density measurements based on VLA observations of the galaxies included in the SFRS. The paper is organized as follows. In Section 2 we describe our sample selection and the analysis procedures used in the present study. Our results are presented and discussed in Section 3. In Section 4 we summarize our main conclusions. Throughout the paper we report median absolute deviations rather than standard deviations, as this statistic is more resilient against outliers in a data set.

\section{Sample and Data Analysis}

In this section we describe the sample selection. We additionally present the VLA observations along with our reduction and imaging procedures, and provide a description of the ancillary data utilized for the present study.

\subsection{Sample Selection}

The SFRS sample comprises nuclear and extranuclear starforming regions in 56 nearby galaxies $(d<30 \mathrm{Mpc})$ observed as part of the SINGS (Kennicutt et al. 2003) and KINGFISH (Kennicutt et al. 2011) legacy programs. Each of these nuclear and extranuclear star-forming complexes have mid-infrared [i.e., low resolution from 5 to $14 \mu \mathrm{m}(0.3 \times 0.9)$ and high resolution from 10 to $37 \mu \mathrm{m}(0 ! 3 \times 0$ !. $)]$ spectral mappings carried out by the IRS instrument on board Spitzer, and $47^{\prime \prime} \times 47^{\prime \prime}$ Herschel/PACS far-infrared spectral mappings for a combination of the principal atomic ISM cooling lines of [O I]63 $\mu \mathrm{m},[\mathrm{O}$ III] $88 \mu \mathrm{m},[\mathrm{N} \mathrm{II}] 122,205 \mu \mathrm{m}$, and [C II]158 $\mu \mathrm{m}$. NGC 5194 and NGC 2403 are exceptions; these galaxies were part of the SINGS sample, but are not formally included in KINGFISH. They were observed with Herschel as part of the Very Nearby Galaxy Survey (VNGS; PI: C. Wilson). Similarly, there are additional KINGFISH galaxies that were not part of SINGS, but have existing Spitzer data: NGC 5457 (M101), IC 342, NGC 3077, and NGC 2146.

SINGS and KINGFISH galaxies were chosen to cover the full range of integrated properties and ISM conditions found in the local universe, spanning the full range in morphological types, a factor of $\sim 10^{5}$ in infrared (IR: $8-1000 \mu \mathrm{m}$ ) luminosity, a factor of $\sim 10^{3}$ in $L_{\mathrm{IR}} / L_{\mathrm{opt}}$, and a large range in SFR $\left(\lesssim 10^{-3}-10 M_{\odot} \mathrm{yr}^{-1}\right)$. Similarly, spectroscopically targeted extranuclear sources included in SINGS and KINGFISH were selected to cover the full range of physical conditions and spectral characteristics found in (bright) infrared sources in nearby galaxies, requiring optical and infrared selections. Optically selected extranuclear regions were chosen to span a large range in physical properties, including the extinction-corrected production rate of ionizing photons $\left[Q\left(H^{0}\right) \sim 10^{49}-10^{52} \mathrm{~s}^{-1}\right]$, metallicity $\left(\sim 0.1-3 Z_{\odot}\right)$, visual extinction $\left(A_{V} \lesssim 4\right.$ mag), radiation field intensity (100-fold range), ionizing stellar temperature $\left[T_{\text {eff }} \sim(3.5-5.5) \times 10^{4} \mathrm{~K}\right]$, and local $\mathrm{H}_{2} / \mathrm{H}$ I ratios $(\lesssim 0.1-\gtrsim 10)$. A sub-sample of infrared-selected extranuclear targets were chosen to span a range in $f_{\nu}(8 \mu \mathrm{m}) / f_{\nu}(24 \mu \mathrm{m})$ and $f_{\mathrm{H} \alpha} / f_{\nu}(8 \mu \mathrm{m})$ ratios.

The total set of observations over the entire sky consists of 118 star-forming complexes (56 nuclei and 62 extranuclear regions), 112 of which (50 nuclei and 62 extranuclear regions; see Tables 1 and 2, respectively) are observable with the VLA (i.e., having $\delta>-35^{\circ}$ ). The coordinates given in both tables are the VLA pointing centers, which correspond to the centers of the Spitzer mid-infrared and Herschel far-infrared spectral line maps. Galaxy morphologies, adopted distances, optically defined nuclear types, diameters $\left(D_{25}\right)$, inclinations $(i)$, and position angles (P.A.) are given in Table 1 . When categorizing nuclear types using Ho et al. (1997), we assign them to be starforming (SF) if they were given an $\mathrm{H}$ II classification or AGN if they were given either a Seyfert or LINER classification. Galaxy morphologies, diameters, and position angles were taken from the Third Reference Catalog of Bright Galaxies (RC3; de Vaucouleurs et al. 1991). For a number of sources, position angles were not given in the RC3 catalog, so we instead use those derived using $2.2 \mu \mathrm{m}$ ( $K_{s}$ band) photometry from the Two Micron All Sky Survey (2MASS) and given in Jarrett et al. (2003). These sources are identified in Table 1. We calculate inclinations using the method described by Dale et al. (1997) such that,

$$
\cos ^{2} i=\frac{(b / a)^{2}-(b / a)_{\mathrm{int}}^{2}}{1-(b / a)_{\mathrm{int}}^{2}},
$$


Table 1

Galaxy Properties and Nuclear Source Positions

\begin{tabular}{|c|c|c|c|c|c|c|c|c|}
\hline Galaxy & $\begin{array}{c}\text { R.A. } \\
(\mathrm{J} 2000)\end{array}$ & $\begin{array}{c}\text { Decl. } \\
(\mathrm{J} 2000)\end{array}$ & Type $^{\mathrm{a}}$ & $\begin{array}{l}\text { Dist. }^{b} \\
(\mathrm{Mpc})\end{array}$ & Nuc. Type & $\begin{array}{c}D_{25}{ }^{\mathrm{a}} \\
(\operatorname{arcmin})\end{array}$ & $\begin{array}{c}i \\
\left({ }^{\circ}\right)\end{array}$ & $\begin{array}{c}\text { P.A. }{ }^{a} \\
\left({ }^{\circ}\right)\end{array}$ \\
\hline NGC 0337 & 005950.3 & -073444 & SBd & 19.3 & SF & $2.9 \times 1.8$ & 52 & 130 \\
\hline NGC 0628 & 013641.7 & +154659 & SAc & 7.2 & $\ldots$ & $10.5 \times 9.5$ & 25 & 25 \\
\hline NGC 0855 & 021403.7 & +275238 & $\mathrm{E}$ & 9.73 & SF & $2.6 \times 1.0$ & 70 & $67^{\mathrm{d}}$ \\
\hline NGC 0925 & 022717.0 & +333443 & SABd & 9.12 & SF & $10.5 \times 5.9$ & 57 & 102 \\
\hline NGC 1097 & 024619.1 & -301628 & $\mathrm{SBb}$ & 14.2 & $\mathrm{AGN}$ & $9.3 \times 6.3$ & 48 & 130 \\
\hline NGC 1266 & 031600.8 & -022538 & SB0 & 30.6 & $\mathrm{AGN}$ & $1.5 \times 1.0$ & 49 & $108^{d}$ \\
\hline NGC 1377 & 033638.9 & -205406 & So & 24.6 & $\ldots$ & $1.8 \times 0.9$ & 61 & 92 \\
\hline IC 0342 & 034648.5 & +680546 & SABcd & 3.28 & $\mathrm{SF}\left(^{*}\right)$ & $21.4 \times 20$ & 21 & $153^{\mathrm{d}}$ \\
\hline NGC 1482 & 035439.5 & -203007 & SA0 & 22.6 & $\mathrm{SF}$ & $2.5 \times 1.4$ & 57 & 103 \\
\hline NGC 2146 & 061837.7 & +782125 & Sbab & 17.2 & $\mathrm{SF}\left(^{*}\right)$ & $6.0 \times 3.4$ & 56 & 57 \\
\hline NGC 2403 & 073650.0 & +653604 & SABcd & 3.22 & $\mathrm{SF}\left({ }^{*}\right)$ & $21.9 \times 12.3$ & 57 & 128 \\
\hline Holmberg II & $\begin{array}{lll}08 & 19 & 13.3\end{array}$ & +704308 & $\mathrm{Im}$ & 3.05 & $\ldots$ & $7.9 \times 6.3$ & 37 & 16 \\
\hline NGC 2798 & 091722.8 & +415958 & $\mathrm{SBa}$ & 25.8 & $\mathrm{SF} / \mathrm{AGN}$ & $2.6 \times 1.0$ & 70 & 160 \\
\hline NGC 2841 & 092202.7 & +505836 & $\mathrm{SAb}$ & 14.1 & $\mathrm{AGN}$ & $8.1 \times 3.5$ & 66 & 147 \\
\hline NGC 2976 & 094715.3 & +675500 & SAc & 3.55 & $\mathrm{SF}$ & $5.9 \times 2.7$ & 64 & 143 \\
\hline NGC 3049 & 095449.6 & +091617 & SBab & 19.2 & $\mathrm{SF}$ & $2.2 \times 1.4$ & 51 & 25 \\
\hline NGC 3077 & 100319.1 & +684402 & I0pec & 3.83 & $\mathrm{SF}\left(^{*}\right)$ & $5.4 \times 4.5$ & 34 & 45 \\
\hline NGC 3190 & 101805.6 & +214955 & SAap & 19.3 & $\mathrm{AGN}\left({ }^{*}\right)$ & $4.4 \times 1.5$ & 73 & 125 \\
\hline NGC 3184 & 101816.7 & +412527 & SABcd & 11.7 & SF & $7.4 \times 6.9$ & 21 & 135 \\
\hline NGC 3198 & 101954.9 & +453259 & $\mathrm{SBc}$ & 14.1 & $\mathrm{SF}$ & $8.5 \times 3.3$ & 68 & 35 \\
\hline IC 2574 & 102848.4 & +682802 & $\mathrm{SABm}$ & 3.79 & $\mathrm{SF}\left(^{*}\right)$ & $13.2 \times 5.4$ & 67 & 50 \\
\hline NGC 3265 & 103106.7 & +284748 & E & 19.6 & $\mathrm{SF}$ & $1.3 \times 1.0$ & 39 & 73 \\
\hline NGC 3351 & 104357.8 & +114214 & $\mathrm{SBb}$ & 9.33 & SF & $7.4 \times 5.0$ & 48 & 13 \\
\hline NGC 3521 & 110548.9 & -000206 & $\mathrm{SABbc}$ & 11.2 & $\mathrm{SF} / \mathrm{AGN}\left({ }^{*}\right)$ & $11.0 \times 5.1$ & 63 & 163 \\
\hline NGC 3621 & 111816.0 & -324842 & SAd & 6.55 & $\mathrm{AGN}$ & $12.3 \times 7.1$ & 55 & 159 \\
\hline NGC 3627 & 112015.0 & +125930 & $\mathrm{SABb}$ & 9.38 & $\mathrm{AGN}$ & $9.1 \times 4.2$ & 64 & 173 \\
\hline NGC 3773 & 113813.0 & +120645 & SA0 & 12.4 & $\mathrm{SF}$ & $1.2 \times 1.0$ & 33 & 165 \\
\hline NGC 3938 & 115249.5 & +440714 & SAc & 17.9 & $\mathrm{SF}\left(^{*}\right)$ & $5.4 \times 4.9$ & 25 & $29^{\mathrm{d}}$ \\
\hline NGC 4254 & 121849.4 & +142459 & SAc & 14.4 & $\mathrm{SF} / \mathrm{AGN}$ & $5.4 \times 4.7$ & 30 & $24^{\mathrm{d}}$ \\
\hline NGC 4321 & 122254.9 & +154921 & SABbc & 14.3 & $\mathrm{AGN}$ & $7.4 \times 6.3$ & 32 & 30 \\
\hline NGC 4536 & 123427.1 & +021117 & SABbc & 14.5 & $\mathrm{SF} / \mathrm{AGN}$ & $7.6 \times 3.2$ & 66 & 130 \\
\hline NGC 4559 & 123557.7 & +275736 & SABcd & 6.98 & $\mathrm{SF}$ & $10.7 \times 4.4$ & 67 & 150 \\
\hline NGC 4569 & 123649.8 & +130946 & SABab & 9.86 & $\mathrm{AGN}$ & $9.5 \times 4.4$ & 64 & 23 \\
\hline NGC 4579 & 123743.6 & +114902 & $\mathrm{SABb}$ & 16.4 & AGN & $5.9 \times 4.7$ & 37 & 95 \\
\hline NGC 4594 & 123959.4 & -113723 & SAa & 9.08 & $\mathrm{AGN}$ & $8.7 \times 3.5$ & 69 & 90 \\
\hline NGC 4625 & 124152.4 & +411624 & SABmp & 9.3 & $\mathrm{SF}$ & $2.2 \times 1.9$ & 31 & $28^{\mathrm{d}}$ \\
\hline NGC 4631 & 124205.9 & +323222 & SBd & 7.62 & $\mathrm{SF}\left(^{*}\right)$ & $15.5 \times 2.7$ & 83 & 86 \\
\hline NGC 4725 & 125026.6 & +253006 & $\mathrm{SABab}$ & 11.9 & $\mathrm{AGN}$ & $10.7 \times 7.6$ & 45 & 35 \\
\hline NGC 4736 & 125053.0 & +410714 & SAab & 4.66 & $\mathrm{AGN}\left({ }^{*}\right)$ & $11.2 \times 9.1$ & 35 & 105 \\
\hline NGC 4826 & 125643.9 & +214100 & SAab & 5.27 & AGN & $10.0 \times 5.4$ & 59 & 115 \\
\hline NGC 5055 & 131549.2 & +420149 & SAbc & 7.94 & AGN & $12.6 \times 7.2$ & 56 & 105 \\
\hline NGC 5194 & 132952.7 & +471143 & SABbcp & 7.62 & $\mathrm{AGN}$ & $11.2 \times 6.9$ & 53 & 163 \\
\hline NGC 5398 & 140120.2 & -330409 & SBdm & 7.66 & $\ldots$ & $2.8 \times 1.7$ & 53 & 172 \\
\hline NGC 5457 & 140312.6 & +542057 & SABcd & 6.7 & $\mathrm{SF}\left(^{*}\right)$ & $28.8 \times 26$ & 26 & $29^{\mathrm{d}}$ \\
\hline NGC 5474 & 140501.3 & +533944 & SAcd & 6.8 & $\mathrm{SF}\left(^{*}\right)$ & $4.8 \times 4.3$ & 27 & $98^{d}$ \\
\hline NGC 5713 & 144011.3 & -001727 & SABbcp & 21.4 & $\mathrm{SF}$ & $2.8 \times 2.5$ & 27 & 10 \\
\hline NGC 5866 & 150629.5 & +554548 & S0 & 15.3 & $\mathrm{AGN}$ & $4.7 \times 1.9$ & 69 & 128 \\
\hline NGC 6946 & 203452.3 & +600914 & SABcd & 6.8 & $\mathrm{SF}$ & $11.5 \times 9.8$ & 32 & $53^{d}$ \\
\hline NGC 7331 & 223704.1 & +342456 & $\mathrm{SAb}$ & 14.5 & AGN & $10.5 \times 3.7$ & 72 & 171 \\
\hline NGC 7793 & 235749.2 & -323524 & SAd & 3.91 & $\mathrm{SF}$ & $9.3 \times 6.3$ & 48 & 98 \\
\hline
\end{tabular}

Notes.

${ }^{a}$ Morphological types, diameters, and position angles were taken from the Third Reference Catalog of Bright Galaxies (RC3; de Vaucouleurs et al. 1991).

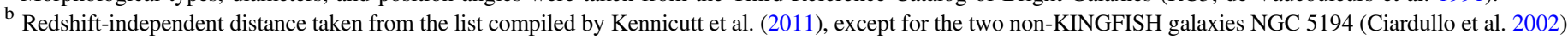
and NGC 2403 (Freedman et al. 2001).

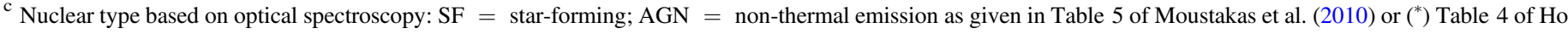
et al. (1997).

${ }^{\mathrm{d}}$ Position angle taken from Jarrett et al. (2003).

where $a$ and $b$ are the observed semimajor and semiminor axes and the disks are oblate spheroids with an intrinsic axial ratio $(b / a)_{\mathrm{int}} \simeq 0.2$ for morphological types earlier than $\mathrm{Sbc}$ and $(b / a)_{\text {int }} \simeq 0.13$ otherwise.

\section{2. $33 \mathrm{GHz}$ VLA Observations and Data Reduction}

Observations in the Ka-band $(26.5-40 \mathrm{GHz})$ were taken during two separate VLA D-configuration cycles. As with our 
Table 2

Extranuclear Source Positions

\begin{tabular}{|c|c|c|}
\hline ID & $\begin{array}{l}\text { R.A. } \\
\text { (J2000) }\end{array}$ & $\begin{array}{l}\text { Decl. } \\
\text { (J2000) }\end{array}$ \\
\hline NGC 0628 Enuc. 1 & 013645.1 & +154751 \\
\hline NGC 0628 Enuc. 2 & 013637.5 & +154512 \\
\hline NGC 0628 Enuc. 3 & 013638.8 & +154425 \\
\hline NGC 0628 Enuc. 4 & 013635.5 & +155011 \\
\hline NGC 1097 Enuc. 1 & 024623.9 & -301750 \\
\hline NGC 1097 Enuc. 2 & 024614.4 & -301504 \\
\hline NGC 2403 Enuc. 1 & 073645.5 & +653700 \\
\hline NGC 2403 Enuc. 2 & 073652.7 & +653646 \\
\hline NGC 2403 Enuc. 3 & 073706.9 & +653639 \\
\hline NGC 2403 Enuc. 4 & 073717.9 & +653346 \\
\hline NGC 2403 Enuc. 5 & 073619.5 & +653704 \\
\hline NGC 2403 Enuc. 6 & 073628.5 & +653350 \\
\hline NGC 2976 Enuc. 1 & 094707.8 & +675552 \\
\hline NGC 2976 Enuc. 2 & 094724.1 & +675356 \\
\hline NGC 3521 Enuc. 1 & 110546.3 & -000409 \\
\hline NGC 3521 Enuc. 2 & 110549.9 & -000339 \\
\hline NGC 3521 Enuc. 3 & 110547.6 & +000033 \\
\hline NGC 3627 Enuc. 1 & 112016.2 & +125750 \\
\hline NGC 3627 Enuc. 2 & 112016.3 & +125844 \\
\hline NGC 3627 Enuc. 3 & 112016.0 & +125952 \\
\hline NGC 3938 Enuc. 1 & 115246.4 & +440701 \\
\hline NGC 3938 Enuc. 2 & 115300.0 & +440755 \\
\hline NGC 4254 Enuc. 1 & 121849.1 & +142359 \\
\hline NGC 4254 Enuc. 2 & 121844.6 & +142425 \\
\hline NGC 4321 Enuc. 1 & 122258.9 & +154935 \\
\hline NGC 4321 Enuc. 2 & 122249.8 & +155029 \\
\hline NGC 4631 Enuc. 1 & 124140.8 & +323151 \\
\hline NGC 4631 Enuc. 2 & 124221.3 & +323306 \\
\hline NGC 4736 Enuc. 1 & 125056.2 & +410720 \\
\hline NGC 5055 Enuc. 1 & 131558.0 & +420026 \\
\hline NGC 5194 Enuc. 1 & 132953.1 & +471240 \\
\hline NGC 5194 Enuc. 2 & 132944.1 & +471021 \\
\hline NGC 5194 Enuc. 3 & 132944.6 & +470955 \\
\hline NGC 5194 Enuc. 4 & 132956.2 & +471407 \\
\hline NGC 5194 Enuc. 5 & 132959.6 & +471401 \\
\hline NGC 5194 Enuc. 6 & 132939.5 & +470835 \\
\hline NGC 5194 Enuc. 7 & 133002.5 & +470952 \\
\hline NGC 5194 Enuc. 8 & 133001.6 & +471252 \\
\hline NGC 5194 Enuc. 9 & 132959.9 & +471112 \\
\hline NGC 5194 Enuc. 10 & 132956.7 & +471046 \\
\hline NGC 5194 Enuc. 11 & 132949.7 & +471329 \\
\hline NGC 5457 Enuc. 1 & 140310.2 & +542057 \\
\hline NGC 5457 Enuc. 2 & 140255.0 & +542226 \\
\hline NGC 5457 Enuc. 3 & 140341.3 & +541904 \\
\hline NGC 5457 Enuc. 4 & 140353.1 & +542206 \\
\hline NGC 5457 Enuc. 5 & 140301.1 & +541428 \\
\hline NGC 5457 Enuc. 6 & 140228.1 & +541626 \\
\hline NGC 5457 Enuc. 7 & 140429.3 & +542346 \\
\hline NGC 5713 Enuc. 1 & 144012.1 & -001747 \\
\hline NGC 5713 Enuc. 2 & 144010.5 & -001747 \\
\hline NGC 6946 Enuc. 1 & 203516.6 & +601057 \\
\hline NGC 6946 Enuc. 2 & 203525.1 & +601003 \\
\hline NGC 6946 Enuc. 3 & 203452.2 & +601241 \\
\hline NGC 6946 Enuc. 4 & 203419.4 & +601009 \\
\hline NGC 6946 Enuc. 5 & 203439.0 & +600453 \\
\hline NGC 6946 Enuc. 6 & 203506.0 & +601100 \\
\hline NGC 6946 Enuc. 7 & 203511.2 & +600859 \\
\hline NGC 6946 Enuc. 8 & 203432.2 & +601019 \\
\hline NGC 6946 Enuc. 9 & 203512.7 & +600852 \\
\hline NGC 7793 Enuc. 1 & 235748.8 & -323658 \\
\hline NGC 7793 Enuc. 2 & 235756.1 & -323540 \\
\hline NGC 7793 Enuc. 3 & 235748.8 & -323452 \\
\hline
\end{tabular}

GBT program, the observing strategy was constructed to make the most efficient use of the telescope. Thus, given the large range in brightness among our targeted regions, we varied the time spent on source based on an estimate of the expected $33 \mathrm{GHz}$ flux density using the Spitzer $24 \mu \mathrm{m}$ maps.

D-configuration observations were obtained in 2011 November (VLA/11B-032) and 2013 March (VLA/13A-129). For the first round of observations, the 8-bit samplers were used, yielding $2 \mathrm{GHz}$ of simultaneous bandwidth, which we used to center $1 \mathrm{GHz}$ wide basebands at 32.5 and $33.5 \mathrm{GHz}$. For the latter run, the 3-bit samplers became available, yielding $8 \mathrm{GHz}$ of instantaneous bandwidth in $2 \mathrm{GHz}$ wide basebands centered at 30, 32, 34, and $36 \mathrm{GHz}$. The standard VLA flux density calibrators 3C 48, 3C 286, and 3C 147 were used.

During the $11 \mathrm{~B}$ semester, there was a correlator malfunction such that for all correlator integration times, only the first second was recorded. In our case, we used a $3 \mathrm{~s}$ dump time, resulting in only obtaining $\frac{1}{3}$ of the requested data. Because of this, a fraction of sources included in VLA/11B-032 were re-observed later in the semester, some of which were observed during the move in DnC-configuration. We additionally re-observed a number of sources during 13A that were not re-observed in 11B. These various cases are identified in Tables 3 and 4

To reduce the VLA data, we used a number of Common Astronomy Software Applications (CASA; McMullin et al. 2007) versions and followed standard calibration and editing procedures, including the utilization of the VLA calibration pipeline. For data calibrated with the VLA pipeline using CASA 4.4.0 or later, we inspected the visibilities and calibration tables for evidence of bad antennas, frequency ranges, and time ranges, flagging correspondingly. We also flagged any instances of RFI, which we found very little of at $33 \mathrm{GHz}$. After flagging, we re-ran the pipeline, and repeated this process until all bad data was removed.

For data calibrated without the pipeline, we used the following general procedure, and regenerated all previous calibration tables as necessary if antennas, frequencies, or time ranges were flagged for having bad data:

1. Generate initial calibration tables for antenna position, opacity, and gain curve.

2. Set the flux calibrator's flux scale using the 2010 version of the Perley \& Butler model.

3. Generate the initial delay calibration table (using the flux calibrator), applying prior calibration tables on-the-fly.

4. Generate the initial short (15 s) integration phase-only gain calibration table (using the flux calibrator), applying the delay table and prior tables on-the-fly.

5. Generate the initial bandpass calibration (using the flux calibrator), applying the delay, phase, and prior tables onthe-fly.

6. Generate the final short integration phase-only gain calibration tables for all calibrators (flux and phase), applying the delay, bandpass, and prior tables on-the-fly.

7. Generate amplitude+phase gain calibration tables for all calibrators, applying the delay, short phase, bandpass, and prior tables on-the-fly.

8. Use the amplitude+phase calibration tables to set the final flux scale calibration for all phase calibrators. 
Table 3

Nuclear Source Imaging Characteristics

\begin{tabular}{|c|c|c|c|c|}
\hline Galaxy & Program ID & $\begin{array}{c}\text { Synthesized } \\
\text { Beam }\end{array}$ & $\begin{array}{c}\sigma \\
\left(\mu \mathrm{Jy} \mathrm{bm}^{-1}\right)\end{array}$ & $\begin{array}{c}\sigma_{T_{\mathrm{b}}} \\
(\mathrm{mK})\end{array}$ \\
\hline NGC 0337 & $\mathrm{VLA} / 11 \mathrm{~B}-32^{\mathrm{a}}$ & 2 !" $04 \times 11^{\prime \prime} 13$ & 12.5 & 6.05 \\
\hline NGC 0628 & $\mathrm{VLA} / 11 \mathrm{~B}-32^{\mathrm{b}}$ & 2 ". $14 \times 1 . " 94$ & 21.1 & 5.66 \\
\hline NGC 0855 & $\mathrm{VLA} / 11 \mathrm{~B}-32^{\mathrm{b}}$ & $1{ }^{\prime \prime} 92 \times 0 . " 93$ & 11.3 & 7.12 \\
\hline NGC 0925 & $\mathrm{VLA} / 11 \mathrm{~B}-32^{\mathrm{b}}$ & 1 1" $91 \times 1 . " 36$ & 12.9 & 5.56 \\
\hline NGC 1097 & $\mathrm{VLA} / 11 \mathrm{~B}-32^{\mathrm{a}}$ & 3 ". $17 \times 1$ ․ 55 & 43.1 & 9.80 \\
\hline NGC 1266 & $\mathrm{VLA} / 11 \mathrm{~B}-32^{\mathrm{b}}$ & $2 ! .42 \times 1{ }^{\prime \prime} 88$ & 52.2 & 12.82 \\
\hline NGC 1377 & $\mathrm{VLA} / 11 \mathrm{~B}-32^{\mathrm{b}}$ & 3 ". $57 \times 1$ ×. 91 & 29.8 & 4.87 \\
\hline IC 0342 & $\mathrm{VLA} / 11 \mathrm{~B}-32^{\mathrm{a}}$ & 1 1! $75 \times 1 . \prime 72$ & 34.6 & 12.78 \\
\hline NGC 1482 & $\mathrm{VLA} / 11 \mathrm{~B}-32^{\mathrm{b}}$ & 3 ". $27 \times 1$ 1" 79 & 72.0 & 13.78 \\
\hline NGC 2146 & VLA $/ 11 B-32^{a}$ & $1{ }^{\prime \prime} 90 \times 1 . " 07$ & 34.1 & 18.79 \\
\hline NGC 2403 & VLA/13A-129 & $2 ! " 42 \times 1$ " 85 & 9.9 & 2.47 \\
\hline Holmberg II & $\mathrm{VLA} / 11 \mathrm{~B}-32^{\mathrm{a}}$ & 1 ". $84 \times 1 . " 01$ & 14.9 & 8.92 \\
\hline NGC 2798 & $\mathrm{VLA} / 11 \mathrm{~B}-32^{\mathrm{b}}$ & $2 ! .07 \times 1 . " 74$ & 18.1 & 5.63 \\
\hline NGC 2841 & $\mathrm{VLA} / 11 \mathrm{~B}-32^{\mathrm{b}}$ & $2 ! .08 \times 1 . " 89$ & 10.1 & 2.85 \\
\hline NGC 2976 & $\mathrm{VLA} / 11 \mathrm{~B}-32^{\mathrm{a}}$ & $2 ! " 40 \times 1 . " 68$ & 19.7 & 5.46 \\
\hline NGC 3049 & VLA $/ 11 B-32,{ }^{b}$ VLA $/ 13 A-129$ & 2 ". $50 \times 2 . " 02$ & 17.9 & 3.95 \\
\hline NGC 3077 & VLA $/ 11 \mathrm{~B}-32^{\mathrm{a}}$ & 2 ". $45 \times 1 . " 66$ & 29.3 & 8.05 \\
\hline NGC 3190 & VLA $/ 11 B-32,{ }^{b}$ VLA $/ 13 A-129$ & 2 ". $13 \times 1 . " 85$ & 13.7 & 3.86 \\
\hline NGC 3184 & VLA/11B-32, ${ }^{\mathrm{b}}$ VLA/13A-129 & $2 . " 51 \times 1 . " 93$ & 13.1 & 3.02 \\
\hline NGC 3198 & $\mathrm{VLA} / 11 \mathrm{~B}-32^{\mathrm{b}}$ & $2 ! .06 \times 1 . ! 98$ & 18.7 & 5.11 \\
\hline IC 2574 & $\mathrm{VLA} / 11 \mathrm{~B}-32^{\mathrm{a}}$ & 2 ". $17 \times 1$ ×. 64 & 15.7 & 4.92 \\
\hline NGC 3265 & VLA/11B-32, ${ }^{\mathrm{b}}$ VLA/13A-129 & 2 ". $15 \times 1{ }^{\prime \prime} 94$ & 12.9 & 3.44 \\
\hline NGC 3351 & VLA $/ 11 B-32,{ }^{b}$ VLA/13A-129 & $2 ! .27 \times 2 ! .04$ & 17.6 & 4.24 \\
\hline NGC 3521 & VLA $/ 11 \mathrm{~B}-32^{\mathrm{b}}$ & $4 . .20 \times 1{ }^{\prime \prime} 98$ & 27.3 & 3.66 \\
\hline NGC 3621 & $\mathrm{VLA} / 11 \mathrm{~B}-32^{\mathrm{b}}$ & $4 . " 32 \times 1 . .58$ & 30.3 & 4.96 \\
\hline NGC 3627 & VLA $/ 11 B-32,{ }^{b}$ VLA/13A-129 & $2 ! \prime 83 \times 1 . ! 83$ & 23.7 & 5.10 \\
\hline NGC 3773 & VLA/11B-32, ${ }^{\mathrm{b}}$ VLA $/ 13 \mathrm{~A}-129$ & $2.99 \times 2 . .50$ & 20.3 & 3.03 \\
\hline NGC 3938 & VLA $/ 11 \mathrm{~B}-32^{\mathrm{a}}$ & $2 . \prime 25 \times 1 . .83$ & 16.5 & 4.47 \\
\hline NGC 4254 & VLA/13A-129 & $2 ! 34 \times 1 !$ × 90 & 15.4 & 3.87 \\
\hline NGC 4321 & VLA/13A-129 & $2 ! 41 \times 1 " .77$ & 18.4 & 4.83 \\
\hline NGC 4536 & VLA/13A-129 & 2 ". $36 \times 2$ "! 16 & 17.4 & 3.79 \\
\hline NGC 4559 & VLA/13A-129 & 3.! $02 \times 1$ ×. 94 & 11.1 & 2.11 \\
\hline NGC 4569 & VLA/13A-129 & $2 ! 41 \times 1 . ! 76$ & 21.7 & 5.70 \\
\hline NGC 4579 & VLA/13A-129 & $2.48 \times 1.78$ & 34.2 & 8.65 \\
\hline NGC 4594 & VLA/13A-129 & 2 ". $98 \times 2$ ". 12 & 20.2 & 3.57 \\
\hline NGC 4625 & VLA/13A-129 & $2 ! .96 \times 2$ ". 09 & 9.2 & 1.66 \\
\hline NGC 4631 & VLA/13A-129 & $2 ! .33 \times 1 . ! 97$ & 15.7 & 3.82 \\
\hline NGC 4725 & VLA/13A-129 & $2 ! .89 \times 1 . ! 97$ & 10.7 & 2.09 \\
\hline NGC 4736 & VLA/13A-129 & $2 ! .98 \times 2$ ". 09 & 20.5 & 3.68 \\
\hline NGC 4826 & VLA/13A-129 & 2 !" $16 \times 1$ ×. 98 & 14.0 & 3.64 \\
\hline NGC 5055 & VLA/13A-129 & $2 ! .76 \times 2$ "! 12 & 16.9 & 3.23 \\
\hline NGC 5194 & VLA/13A-129 & $2 ! .27 \times 1 . ! 80$ & 13.6 & 3.72 \\
\hline NGC 5398 & VLA/13A-129 & $5 . " 42 \times 1.79$ & 18.1 & 2.08 \\
\hline NGC 5457 & VLA/13A-129 & $2 ! 36 \times 1 . .76$ & 14.1 & 3.79 \\
\hline NGC 5474 & VLA/13A-129 & 2 ". $18 \times 1{ }^{\prime \prime} 84$ & 9.4 & 2.61 \\
\hline NGC 5713 & VLA/13A-129 & 2 !" $39 \times 2$ "! 14 & 14.5 & 3.17 \\
\hline NGC 5866 & VLA/13A-129 & $2 ! .28 \times 1 . ! 79$ & 16.2 & 4.43 \\
\hline NGC 6946 & $\mathrm{VLA} / 11 \mathrm{~B}-32^{\mathrm{a}}$ & $2 ! \prime 12 \times 1{ }^{\prime \prime} 70$ & 31.5 & 9.72 \\
\hline NGC 7331 & VLA $/ 11 \mathrm{~B}-32^{\mathrm{b}}$ & $3 ! .02 \times 1 . ! 88$ & 35.8 & 7.04 \\
\hline NGC 7793 & VLA $/ 11 \mathrm{~B}-32^{\mathrm{b}}$ & 4 ." $48 \times 1$ 1. 69 & 23.6 & 3.48 \\
\hline
\end{tabular}

Notes. VLA11B-32 observations were conducted between October 2011 and January 2012. VLA/13A-129 observations were conducted between Februrary and March 2013. a Original observations suffered from the " $1 \mathrm{~s}$ " WIDAR correlator malfunction, but the source was later reobserved for the nominal integration time.

b Observations suffered from the " $1 \mathrm{~s}$ " WIDAR correlator malfunction, leading to only $\frac{1}{3}$ of the integration time being recorded.

9. Generate the final long (full scan) integration phase gain calibrations for all calibrators, applying the delay, bandpass, flux scale, and prior tables on-the-fly.
10. Apply the long integration phase, bandpass, delay, flux scale, and prior tables to all science targets.

For all delay and bandpass tables applied on-the-fly, we used the default nearest-neighbor interpolation. For phase and flux scale tables, we used a linear interpolation.

For all 87 nuclear and extranuclear regions that were calibrated by hand using CASA versions 4.2.1 or earlier, the 2010 Perley \& Butler flux density scale was applied as the default. This is different from the flux density scale used in the pipeline-calibrated data run with CASA version 4.4.0 or later (i.e., Perley \& Butler 2013). To place everything on the same flux density scale, we corrected the amplitude of the final images for all 87 regions by multiplying them by the ratio of the Perley \& Butler 2013 to 2010 flux density scalings. The average correction factor was near unity at 0.98 , with an rms scatter of 0.01 .

\subsection{Interferometric Imaging}

Calibrated VLA measurement sets for each source were imaged using the task TCLEAN in CASA version 4.6.0. For some cases (see Tables 3 and 4), the Ka-band images contain data from observations taken during both the $11 \mathrm{~B}$ and $13 \mathrm{~A}$ semesters, but are heavily weighted by the 13A semester observations, as those include significantly more data. The mode of TCLEAN was set to multi-frequency synthesis (MFS; Conway et al. 1990; Sault \& Wieringa 1994). We chose to use Briggs weighting with ROBUST $=0.5$, and set the variable NTERMS $=2$, which allows the cleaning procedure to also model the spectral index variations on the sky. To help deconvolve extended low-intensity emission, we took advantage of the multiscale clean option (Cornwell 2008; Rau \& Cornwell 2011) in CASA, searching for structures with scales $\approx 1$ and 3 times the FWHM of the synthesized beam. The choice of our final imaging parameters was the result of extensive experimentation to identify values that yielded the best combination of brightness-temperature sensitivity and reduction of artifacts resulting from strong sidelobes in the naturally weighted beam for these snapshot-like observations.

The images were placed on a $512 \times 512$ pixel grid with a pixel scale of 0 ". 3. However, for two sources (NGC 0628 Enuc. 4 and NGC 0855), the pixel scale was reduced to 0 " 15 to ensure that the FWHM of the synthesized beam minor axis remained Nyquist-sampled.

For two sources in the sample, NGC 4594 and NGC 4579, a signal-to-noise ratio $(\mathrm{S} / \mathrm{N}) \geqslant 3$ was achieved across the majority of all channels and spectral windows. This allowed us to accurately perform phase-only, and subsequently amplitude + phase, self-calibration for these two sources. The peak brightness of the self-calibrated images differs from that of the originals by less than 5\%; however, the new peak $\mathrm{S} / \mathrm{Ns}$ of NGC 4594 and NGC 4579 are improved by factors of $\approx 2$ and $\approx 3$, respectively (achieving peak $\mathrm{S} / \mathrm{Ns}$ of $\sim 2900$ and $\sim 1500$, respectively).

A primary beam correction was applied using the CASA task IMPBCOR before analyzing the images. The primary-beamcorrected continuum images at $33 \mathrm{GHz}$ for each target are shown in Figure 1. The FWHMs of the synthesized beams are given in Tables 3 and 4 for all sources, along with the corresponding pointsource and brightness-temperature rms values for each of the final images. Given the range of distances to the sample galaxies, this ensured that the linear scale investigated was always $\lesssim 300$ pc 
Table 4

Extranuclear Source Imaging Characteristics

\begin{tabular}{|c|c|c|c|c|}
\hline Galaxy & Program ID & $\begin{array}{c}\text { Synthesized } \\
\text { Beam }\end{array}$ & $\begin{array}{c}\sigma \\
\left(\mu \mathrm{Jy} \mathrm{bm}^{-1}\right)\end{array}$ & $\begin{array}{c}\sigma_{T_{\mathrm{b}}} \\
(\mathrm{mK})\end{array}$ \\
\hline NGC 0628 Enuc. 1 & VLA $/ 11 \mathrm{~B}-32^{\mathrm{b}}$ & $2 ! .08 \times 1 ! " 92$ & 25.7 & 7.20 \\
\hline NGC 0628 Enuc. 2 & $\mathrm{VLA} / 11 \mathrm{~B}-32^{\mathrm{b}}$ & $2 ! " 04 \times 1 " .88$ & 19.7 & 5.73 \\
\hline NGC 0628 Enuc. 3 & VLA $/ 11 \mathrm{~B}-32^{\mathrm{b}}$ & $2 ! " 05 \times 1 " .80$ & 26.6 & 8.04 \\
\hline NGC 0628 Enuc. 4 & $\mathrm{VLA} / 11 \mathrm{~B}-32^{\mathrm{a}}$ & 1 1! $75 \times 0 . " 94$ & 10.8 & 7.32 \\
\hline NGC 1097 Enuc. 1 & $\mathrm{VLA} / 11 \mathrm{~B}-32^{\mathrm{a}}$ & $2 ! .00 \times 1 ! .71$ & 13.7 & 4.47 \\
\hline NGC 1097 Enuc. 2 & $\mathrm{VLA} / 11 \mathrm{~B}-32^{\mathrm{a}}$ & $2 ! ! 11 \times 1 ! .67$ & 14.1 & 4.48 \\
\hline NGC 2403 Enuc. 1 & VLA/13A-129 & $2 ! .60 \times 1 " ! 80$ & 14.1 & 3.36 \\
\hline NGC 2403 Enuc. 2 & VLA/13A-129 & $2 " .54 \times 1 ! .80$ & 13.8 & 3.38 \\
\hline NGC 2403 Enuc. 3 & VLA/13A-129 & $2 ! .52 \times 1 " .79$ & 18.1 & 4.47 \\
\hline NGC 2403 Enuc. 4 & VLA/13A-129 & $2 ! 44 \times 1 ! " 81$ & 10.0 & 2.53 \\
\hline NGC 2403 Enuc. 5 & VLA/13A-129 & $2 ! .75 \times 1 ! .79$ & 13.9 & 3.15 \\
\hline NGC 2403 Enuc. 6 & VLA/13A-129 & $2 ! .71 \times 1 ! " 83$ & 9.8 & 2.21 \\
\hline NGC 2976 Enuc. 1 & $\mathrm{VLA} / 11 \mathrm{~B}-32^{\mathrm{a}}$ & $2 ! " 39 \times 1$ × 66 & 19.6 & 5.53 \\
\hline NGC 2976 Enuc. 2 & $\mathrm{VLA} / 11 \mathrm{~B}-32^{\mathrm{a}}$ & $2 ! 38 \times 1 ! .71$ & 21.7 & 5.93 \\
\hline NGC 3521 Enuc. 1 & VLA $/ 11 \mathrm{~B}-32^{\mathrm{b}}$ & $4 ! " 01 \times 2 ! " 08$ & 36.8 & 4.94 \\
\hline NGC 3521 Enuc. 2 & VLA $/ 11 \mathrm{~B}-32^{\mathrm{b}}$ & $4 ! .74 \times 1.93$ & 34.3 & 4.18 \\
\hline NGC 3521 Enuc. 3 & VLA $/ 11 \mathrm{~B}-32^{\mathrm{b}}$ & $4 ! " 23 \times 1 "$ " 95 & 26.8 & 3.63 \\
\hline NGC 3627 Enuc. 1 & VLA $/ 11 \mathrm{~B}-32,{ }^{\mathrm{b}}$ VLA/13A-129 & $2 ! 45 \times 2 " .03$ & 19.6 & 4.40 \\
\hline NGC 3627 Enuc. 2 & VLA $/ 11 \mathrm{~B}-32,^{\mathrm{b}}$ VLA/13A-129 & 2 ". $55 \times 2 ! " 08$ & 19.4 & 4.08 \\
\hline NGC 3627 Enuc. 3 & VLA $/ 11 \mathrm{~B}-32{ }^{\mathrm{b}}{ }^{\mathrm{V}} \mathrm{VLA} / 13 \mathrm{~A}-129$ & $2 ! " 43 \times 1 " .93$ & 14.3 & 3.41 \\
\hline NGC 3938 Enuc. 1 & VLA $/ 11 \mathrm{~B}-32^{\mathrm{a}}$ & $2 ! " 34 \times 1 ! .83$ & 19.4 & 5.04 \\
\hline NGC 3938 Enuc. 2 & $\mathrm{VLA} / 11 \mathrm{~B}-32^{\mathrm{a}}$ & $2 ! .22 \times 1 ! .78$ & 21.0 & 5.93 \\
\hline NGC 4254 Enuc. 1 & VLA/13A-129 & $2 ! .35 \times 1 ! 93$ & 16.0 & 3.97 \\
\hline NGC 4254 Enuc. 2 & VLA/13A-129 & $2 ! " 40 \times 1 " .96$ & 10.8 & 2.56 \\
\hline NGC 4321 Enuc. 1 & VLA/13A-129 & $2 ! 34 \times 1 ! .79$ & 12.1 & 3.20 \\
\hline NGC 4321 Enuc. 2 & VLA/13A-129 & $2 ! " 33 \times 1 " .82$ & 12.3 & 3.24 \\
\hline NGC 4631 Enuc. 1 & VLA/13A-129 & $2 ! 42 \times 1 "$ ×99 & 10.6 & 2.46 \\
\hline NGC 4631 Enuc. 2 & VLA/13A-129 & $2 " .23 \times 1 " .98$ & 11.1 & 2.79 \\
\hline NGC 4736 Enuc. 1 & VLA/13A-129 & $2 ! " 89 \times 2$ ". 03 & 17.9 & 3.42 \\
\hline NGC 5055 Enuc. 1 & VLA/13A-129 & $2 ! .81 \times 2 ! .05$ & 15.3 & 2.97 \\
\hline NGC 5194 Enuc. 1 & VLA/13A-129 & $2 ! .12 \times 1 " ! 81$ & 14.1 & 4.11 \\
\hline NGC 5194 Enuc. 2 & VLA/13A-129 & $2 ! .32 \times 1 ! " 80$ & 13.2 & 3.54 \\
\hline NGC 5194 Enuc. 3 & VLA/13A-129 & $2 ! ! 17 \times 1 ! " 80$ & 9.8 & 2.79 \\
\hline NGC 5194 Enuc. 4 & VLA/13A-129 & $2 ! ! 10 \times 1 ! ! 84$ & 10.2 & 2.96 \\
\hline NGC 5194 Enuc. 5 & VLA/13A-129 & $2 . " 37 \times 1 . " 73$ & 19.8 & 5.39 \\
\hline NGC 5194 Enuc. 6 & VLA/13A-129 & $2 ! " 39 \times 1 ! " 82$ & 9.4 & 2.42 \\
\hline NGC 5194 Enuc. 7 & VLA/13A-129 & $2 ! ! 38 \times 1 ! " 80$ & 19.6 & 5.11 \\
\hline NGC 5194 Enuc. 8 & VLA/13A-129 & $2 ! 38 \times 1 ! " 80$ & 18.5 & 4.83 \\
\hline NGC 5194 Enuc. 9 & VLA/13A-129 & $2 ! .38 \times 1 . " 77$ & 20.5 & 5.46 \\
\hline NGC 5194 Enuc. 10 & VLA/13A-129 & $2 ! .07 \times 1 ! " 84$ & 15.4 & 4.52 \\
\hline NGC 5194 Enuc. 11 & VLA/13A-129 & $2 ! ! 14 \times 1 " .81$ & 9.9 & 2.84 \\
\hline NGC 5457 Enuc. 1 & VLA/13A-129 & $2 ! .26 \times 1 " ! 80$ & 8.9 & 2.44 \\
\hline NGC 5457 Enuc. 2 & VLA/13A-129 & $2 ! " 42 \times 2 ! " 33$ & 15.2 & 3.02 \\
\hline NGC 5457 Enuc. 3 & VLA/13A-129 & $2 ! " 37 \times 2 ! " 26$ & 21.9 & 4.57 \\
\hline NGC 5457 Enuc. 4 & VLA/13A-129 & $2 ! " 28 \times 1 ! .78$ & 13.8 & 3.78 \\
\hline NGC 5457 Enuc. 5 & VLA/13A-129 & $2 ! " 43 \times 2$ "! 33 & 14.8 & 2.92 \\
\hline NGC 5457 Enuc. 6 & VLA/13A-129 & $2 ! " 43 \times 2 ! " 34$ & 15.5 & 3.05 \\
\hline NGC 5457 Enuc. 7 & VLA/13A-129 & $2 ! .21 \times 1 ! " 80$ & 13.5 & 3.78 \\
\hline NGC 5713 Enuc. 1 & VLA/13A-129 & $2 ! ! 36 \times 2 ! " 16$ & 10.5 & 2.31 \\
\hline NGC 5713 Enuc. 2 & VLA/13A-129 & $2 ! " 44 \times 2$ !" 15 & 14.3 & 3.03 \\
\hline NGC 6946 Enuc. 1 & $\mathrm{VLA} / 11 \mathrm{~B}-32^{\mathrm{a}}$ & $2 ! .08 \times 1 ! .78$ & 16.2 & 4.87 \\
\hline NGC 6946 Enuc. 2 & $\mathrm{VLA} / 11 \mathrm{~B}-32^{\mathrm{a}}$ & $2 ! ! 13 \times 1 ! .87$ & 17.3 & 4.85 \\
\hline NGC 6946 Enuc. 3 & VLA $/ 11 \mathrm{~B}-32^{\mathrm{a}}$ & $2 ! " 11 \times 1 ! " 86$ & 10.5 & 3.01 \\
\hline NGC 6946 Enuc. 4 & $\mathrm{VLA} / 11 \mathrm{~B}-32^{\mathrm{a}}$ & $2 ! ! 17 \times 1 ! " 82$ & 10.7 & 3.01 \\
\hline NGC 6946 Enuc. 5 & $\mathrm{VLA} / 11 \mathrm{~B}-32^{\mathrm{a}}$ & $2 ! " 08 \times 1 ! " 86$ & 10.8 & 3.12 \\
\hline NGC 6946 Enuc. 6 & $\mathrm{VLA} / 11 \mathrm{~B}-32^{\mathrm{a}}$ & $2 ! .08 \times 1 ! 74$ & 16.3 & 5.05 \\
\hline NGC 6946 Enuc. 7 & VLA/11B-32 ${ }^{\mathrm{a}}$ & $2 ! " 08 \times 1 ! 78$ & 16.1 & 4.86 \\
\hline NGC 6946 Enuc. 8 & $\mathrm{VLA} / 11 \mathrm{~B}-32^{\mathrm{a}}$ & $2 ! ! 14 \times 1 ! .70$ & 16.7 & 5.09 \\
\hline NGC 6946 Enuc. 9 & VLA $/ 11 \mathrm{~B}-32^{\mathrm{a}}$ & $2 ! " 08 \times 1 ! 78$ & 16.1 & 4.86 \\
\hline NGC 7793 Enuc. 1 & VLA $/ 11 \mathrm{~B}-32^{\mathrm{b}}$ & $4 ! .25 \times 1 !$. 64 & 29.8 & 4.78 \\
\hline
\end{tabular}


Table 4

(Continued)

\begin{tabular}{|c|c|c|c|c|}
\hline Galaxy & Program ID & $\begin{array}{c}\text { Synthesized } \\
\text { Beam }\end{array}$ & $\begin{array}{c}\sigma \\
\left(\mu \mathrm{Jy} \mathrm{bm}^{-1}\right)\end{array}$ & $\begin{array}{c}\sigma_{T_{\mathrm{b}}} \\
(\mathrm{mK})\end{array}$ \\
\hline NGC 7793 Enuc. 2 & VLA $/ 11 \mathrm{~B}-32^{\mathrm{b}}$ & $4 ! " 46 \times 1$ × 70 & 22.5 & 3.32 \\
\hline NGC 7793 Enuc. 3 & $\mathrm{VLA} / 11 \mathrm{~B}-32^{\mathrm{b}}$ & $4 " .60 \times 1 " .58$ & 37.0 & 5.67 \\
\hline
\end{tabular}

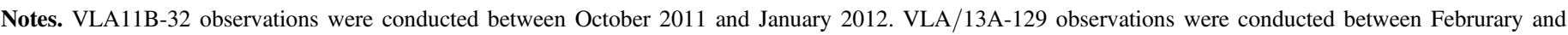
March 2013.

a Original observations suffered from the " $1 \mathrm{~s}$ " WIDAR correlator malfunction, but the source was later reobserved for the nominal integration time.

b Observations suffered from the " $1 \mathrm{~s}$ " WIDAR correlator malfunction, leading to only $\frac{1}{3}$ of the integration time being recorded.

(i.e., the size of giant $\mathrm{H}$ II regions). We also note that the VLA images made with the chosen array configurations should be sensitive to extended emission on angular scales up to $\approx 24^{\prime \prime}$ for these snapshot observations.

We also created a suite of $(u, v)$-tapered images for all nuclear and extranuclear regions in order to assess the potential for missing large-scale emission. After tapering to $2 . " 5$, we find that we recover $\sim 3 \%$ more flux density relative to the nontapered images, suggesting that on the scales of the individual $\mathrm{H}$ II regions and nuclei, we are not missing a significant amount of the source flux density.

\subsection{Ancillary Data}

The $\mathrm{H} \alpha$ imaging used in the analysis is taken from references cited in the compilation by Leroy et al. (2012), where details about the data quality and preparation (e.g., correction for [N II] emission) can be found. $\mathrm{H} \alpha$ images were corrected for foreground stars. The typical resolution of the seeing-limited $\mathrm{H} \alpha$ images is $\approx 1^{\prime \prime}-2^{\prime \prime}$, and the calibration uncertainty among these maps is taken to be $\approx 20 \%$.

Archival Spitzer $24 \mu \mathrm{m}$ data shown in Figure 1 were largely taken from the SINGS and Local Volume Legacy (LVL) legacy programs, and have a calibration uncertainty of $\approx 5 \%$. Details on the associated observation strategies and data reduction steps can be found in Dale et al. (2007, 2009, respectively). Two galaxies, IC 342 and NGC 2146, were not a part of SINGS or LVL; their $24 \mu \mathrm{m}$ imaging comes from Engelbracht et al. (2008).

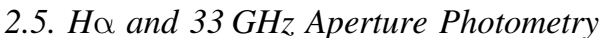

Before making photometric measurements, we aligned the $\mathrm{H} \alpha$ images to the $33 \mathrm{GHz}$ VLA images, which have sub-arcsecond astrometric accuracy. In most cases, the $\mathrm{H} \alpha$ images had existing astrometric solutions matching multiple $\mathrm{H} \alpha$ peaks with $33 \mathrm{GHz}$ counterparts to better than half of the synthesized beam FWHM. We adopted the existing astrometry for these galaxies. For those remaining galaxies with multiple bright radio sources (e.g., NGC 0628), we aligned the $\mathrm{H} \alpha$ images by eye, ensuring that the peaks of multiple bright features matched their radio counterparts within $1^{\prime \prime}$. While there may be physical offsets between $33 \mathrm{GHz}$ and $\mathrm{H} \alpha$ emission arising from high levels of extinction, we note that these offsets are unlikely to be systematic for multiple distinct peaks. In our alignment process, we did not encounter any cases for which the astrometry is significantly affected. ${ }^{11}$ We adopt the

\footnotetext{
11 The nucleus of NGC 4631 is a good example of a case where the $\mathrm{H} \alpha$ and $33 \mathrm{GHz}$ morphologies are clearly distinct. For this galaxy, we note that outside of the $33 \mathrm{GHz}$ field-of-view, the $\mathrm{H} \alpha$ and $24 \mu \mathrm{m}$ images align to better than $1^{\prime \prime}$ and that within the $33 \mathrm{GHz}$ field-of-view, multiple $24 \mu \mathrm{m}$ and $33 \mathrm{GHz}$ peaks align to $\approx 0$ ". 5 . We suspect that the $\mathrm{H} \alpha$ versus $33 \mathrm{GHz}$ mismatches are caused by high extinction along the line-of-sight into this edge-on galaxy.
}

existing astrometry for galaxies with only one detected radio source (e.g., NGC 3198).

Due to the higher intrinsic brightness of the $\mathrm{H} \alpha$ transition relative to free-free emission, our source detection is primarily limited by the $33 \mathrm{GHz}$ noise and brightness-temperature sensitivity given in Tables 3 and 4 . Because of this, we identified photometric regions by drawing rectangular and polygon apertures around strongly detected $33 \mathrm{GHz}$ sources. Using PYBDSM ${ }^{12}$ (Mohan \& Rafferty 2015), we have verified that the native resolution $33 \mathrm{GHz}$ selected sample is complete down to $5 \sigma$ for sources with angular sizes comparable to the $\sim 2^{\prime \prime}$ synthesized beam. To minimize the relative contribution from large angular-scale $\mathrm{H} \alpha$ emission that might fall under the $33 \mathrm{GHz}$ brightness-temperature sensitivity threshold, these apertures are drawn tightly around the brightest parts of the $33 \mathrm{GHz}$ sources. For $33 \mathrm{GHz}$ non-detections, we simply drew a large aperture encompassing $\mathrm{H} \alpha$ (or $24 \mu \mathrm{m}$, see Section 2.6) structures near the phase center. The regions, listed in Table 5, are named according to the nearest $33 \mathrm{GHz}$ image, with an alphabetical suffix if there are multiple regions corresponding to one image. For example, "NGC 2403 Enuc 2. B" is 1 of 3 regions in the image of extranuclear region 2 in NGC 2403. It is also visible in the image of NGC 2403's nucleus, which has only one (non-detection) region: "NGC 2403" (see Figure 1).

Using the CASA task IMSTAT, we measured and report the $\mathrm{H} \alpha$ line flux and $33 \mathrm{GHz}$ flux density for each region in Table 5 detected with an $\mathrm{S} / \mathrm{N}>3$. For sources that are not detected at this significance we provide a corresponding $3 \sigma$ upper limit. The uncertainty in the $33 \mathrm{GHz}$ flux density is taken to be the standard VLA calibration uncertainty $(\sim 3 \%$; Perley \& Butler 2013) added in quadrature with the empirically measured noise from empty regions in each image given in Tables 3 and 4. As stated in Section 2.4, the calibration uncertainty of the $\mathrm{H} \alpha$ narrowband imaging is $\approx 20 \%$, which dominates the uncertainty of the $\mathrm{H} \alpha$ photometry. Also provided in Table 5 is a measure of the galactocentric radius $\left(r_{\mathrm{G}}\right)$ in units of $\mathrm{kpc}$ for each position. These values are calculated using the assumed galaxy inclinations, position angles, and distances listed in Table 1.

\subsection{Inclusion of $24 \mu \mathrm{m}$ Data with Aperture Photometry}

To accurately match the photometry obtained with the $33 \mathrm{GHz}$ and $\mathrm{H} \alpha$ images to that measured using the Spitzer $24 \mu \mathrm{m}$ data, which is at much lower resolution $\left(\approx 7^{\prime \prime}\right)$, we first resolution-matched the images. Both the $33 \mathrm{GHz}$ and $\mathrm{H} \alpha$ images were convolved with a Gaussian kernel resulting in a final FWHM of $7 "$. Following the image registration method

\footnotetext{
12 http://www.astron.nl/citt/pybdsm/
} 

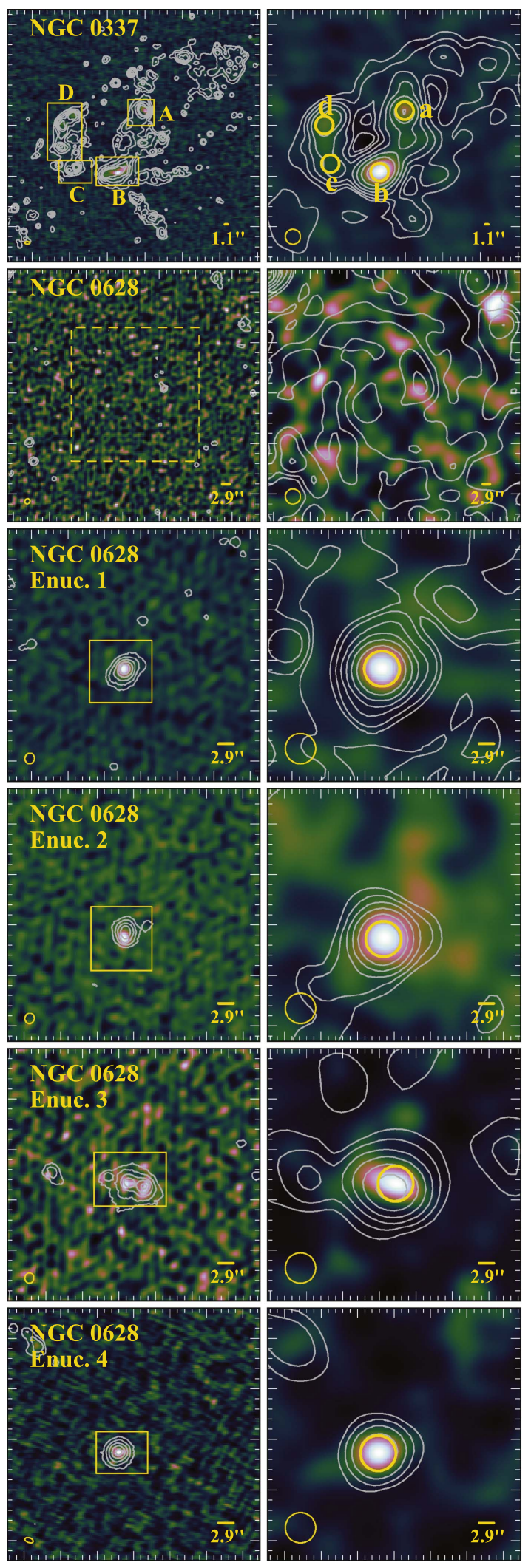

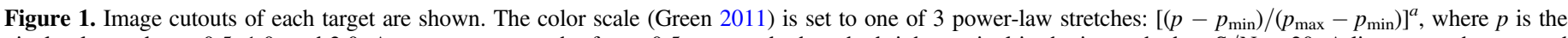

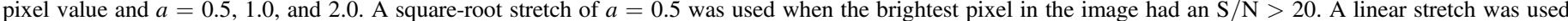

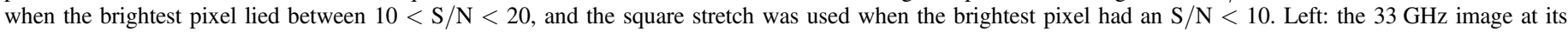

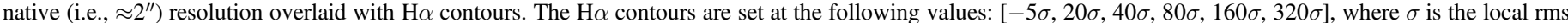

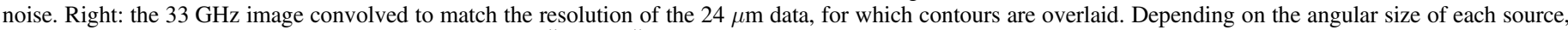

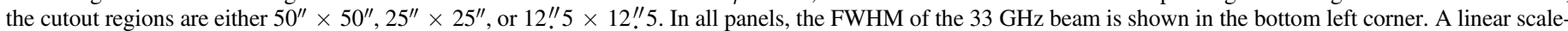

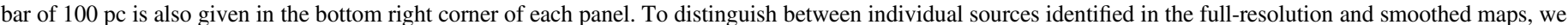

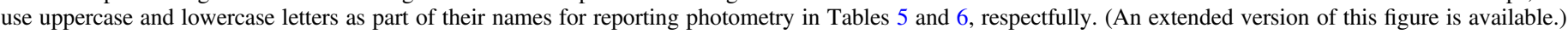


Table 5

Source Photometry

\begin{tabular}{|c|c|c|c|c|c|}
\hline Source ID & $\begin{array}{l}\text { R.A. } \\
(\mathrm{J} 2000)\end{array}$ & $\begin{array}{c}\text { Decl. } \\
(\mathrm{J} 2000)\end{array}$ & $\begin{array}{c}S_{33 \mathrm{GHz}} \\
(\mathrm{mJy})\end{array}$ & $\begin{array}{c}f_{\mathrm{H}_{\alpha}} / 10^{-13} \\
\left(\text { erg s }^{-1} \mathrm{~cm}^{-2}\right)\end{array}$ & $\begin{array}{c}r_{\mathrm{G}} \\
(\mathrm{kpc})\end{array}$ \\
\hline NGC 0337 A & 005950.00 & -073434.7 & $0.57 \pm 0.02$ & $3.40 \pm 0.68$ & 0.823 \\
\hline NGC $0337 \mathrm{~B}$ & 005950.74 & -073458.7 & $1.79 \pm 0.06$ & $6.18 \pm 1.24$ & 2.100 \\
\hline NGC $0337 \mathrm{C}$ & 005951.77 & -073457.2 & $0.15 \pm 0.01$ & $1.29 \pm 0.26$ & 2.946 \\
\hline NGC $0337 \mathrm{D}$ & 005951.87 & -073436.2 & $1.01 \pm 0.03$ & $5.12 \pm 1.02$ & 3.699 \\
\hline NGC 0628 Enuc. 4 & 013635.71 & +155007.2 & $0.26 \pm 0.01$ & $1.15 \pm 0.23$ & 7.611 \\
\hline NGC 0628 Enuc. 2 & 013637.65 & +154507.2 & $0.48 \pm 0.02$ & $1.04 \pm 0.21$ & 4.468 \\
\hline NGC 0628 Enuc. 3 & 013638.92 & +154422.9 & $0.59 \pm 0.03$ & $2.14 \pm 0.43$ & 5.715 \\
\hline NGC 0628 & 013643.26 & +154638.6 & $0.24 \pm 0.02$ & $3.26 \pm 0.65$ & 1.210 \\
\hline NGC 0628 Enuc. 1 & 013645.24 & +154748.0 & $0.64 \pm 0.03$ & $1.74 \pm 0.35$ & 2.463 \\
\hline NGC 0855 & 021403.55 & +275238.9 & $0.87 \pm 0.03$ & $\ldots$ & 0.036 \\
\hline NGC 0925 & 022717.67 & +333428.0 & $0.24 \pm 0.01$ & $8.59 \pm 1.72$ & 1.314 \\
\hline NGC 1097 Enuc. 2 & 024614.28 & -301456.7 & $0.52 \pm 0.02$ & $\ldots$ & 7.990 \\
\hline NGC $1097 \mathrm{~B}$ & 024618.31 & -301631.9 & $17.51 \pm 0.53$ & $\ldots$ & 0.885 \\
\hline NGC 1097 A & 024618.98 & -301629.2 & $3.77 \pm 0.12$ & $\ldots$ & 0.030 \\
\hline NGC 1097 Enuc. 1 & 024621.19 & -301727.1 & $0.69 \pm 0.02$ & $\ldots$ & 4.883 \\
\hline NGC 1266 & 031600.76 & -022538.6 & $10.33 \pm 0.31$ & $\ldots$ & 0.168 \\
\hline NGC 1377 & 033639.11 & -205343.8 & $0.58 \pm 0.03$ & $\ldots$ & 5.952 \\
\hline IC $0342 \mathrm{~A}$ & 034647.80 & +680546.0 & $18.05 \pm 0.54$ & $\ldots$ & 0.066 \\
\hline IC $0342 \mathrm{~B}$ & 034648.61 & +680543.6 & $1.97 \pm 0.07$ & $\ldots$ & 0.039 \\
\hline IC $0342 \mathrm{C}$ & 034649.04 & +680546.0 & $3.87 \pm 0.12$ & $\ldots$ & 0.050 \\
\hline IC 0342 D & 034649.20 & +680549.9 & $2.36 \pm 0.08$ & $\cdots$ & 0.093 \\
\hline NGC 1482 & 035439.07 & -203008.5 & $21.28 \pm 0.64$ & $\ldots$ & 0.272 \\
\hline NGC 2146 A & 061833.93 & +782134.3 & $6.97 \pm 0.21$ & $\ldots$ & 2.122 \\
\hline NGC 2146 B & 061835.22 & +782130.7 & $11.51 \pm 0.35$ & $\ldots$ & 1.348 \\
\hline NGC 2146 C & 061836.61 & +782127.4 & $9.41 \pm 0.28$ & $\ldots$ & 0.581 \\
\hline NGC 2146 D & 061837.50 & +782124.1 & $24.61 \pm 0.74$ & $\ldots$ & 0.105 \\
\hline NGC 2146 E & 061838.79 & +782122.3 & $5.54 \pm 0.17$ & $\ldots$ & 0.615 \\
\hline NGC $2146 \mathrm{~F}$ & 061838.99 & +782119.9 & $5.47 \pm 0.17$ & $\ldots$ & 0.955 \\
\hline NGC $2146 \mathrm{G}$ & 061839.38 & +782118.1 & $7.48 \pm 0.23$ & $\ldots$ & 1.278 \\
\hline NGC 2403 Enuc. 5 A & 073619.69 & +65 3704.9 & $0.98 \pm 0.03$ & $4.39 \pm 0.88$ & 3.484 \\
\hline NGC 2403 Enuc. 5 B & 073620.32 & +653707.3 & $0.84 \pm 0.03$ & $3.67 \pm 0.73$ & 3.396 \\
\hline NGC 2403 Enuc. 6 & 073628.69 & +653348.2 & $0.85 \pm 0.03$ & $3.84 \pm 0.77$ & 5.406 \\
\hline NGC 2403 Enuc. $1 \mathrm{~A}$ & 073642.01 & +653651.6 & $0.25 \pm 0.02$ & $0.54 \pm 0.11$ & 1.085 \\
\hline NGC 2403 Enuc. 1 B & 073645.60 & +65 3701.8 & $1.48 \pm 0.05$ & $8.48 \pm 1.70$ & 1.214 \\
\hline NGC 2403 & 073646.95 & +653543.3 & $0.35 \pm 0.01$ & $9.31 \pm 1.86$ & 0.802 \\
\hline NGC 2403 Enuc. 2 A & 073649.16 & +653652.0 & $0.51 \pm 0.02$ & $2.30 \pm 0.46$ & 1.123 \\
\hline NGC 2403 Enuc. 2 B & 073652.12 & +653648.4 & $0.65 \pm 0.02$ & $2.91 \pm 0.58$ & 1.263 \\
\hline NGC 2403 Enuc. 2 C & 073652.65 & +653646.9 & $0.54 \pm 0.02$ & $2.56 \pm 0.51$ & 1.276 \\
\hline NGC 2403 Enuc. 3 A & 073705.21 & +653641.7 & $0.63 \pm 0.03$ & $2.16 \pm 0.43$ & 2.637 \\
\hline NGC 2403 Enuc. 3 B & 073706.85 & +653638.7 & $3.56 \pm 0.11$ & $12.79 \pm 2.56$ & 2.793 \\
\hline NGC 2403 Enuc. 3 C & 073707.92 & +653638.1 & $2.03 \pm 0.06$ & $5.34 \pm 1.07$ & 2.920 \\
\hline NGC 2403 Enuc. 4 & 073718.14 & +65 3346.9 & $0.99 \pm 0.03$ & $3.26 \pm 0.65$ & 3.463 \\
\hline Holmberg II & 081913.12 & +704308.6 & $0.78 \pm 0.03$ & $5.44 \pm 1.09$ & 0.742 \\
\hline NGC 2798 & 091722.83 & +420001.3 & $4.60 \pm 0.14$ & $10.83 \pm 2.17$ & 0.221 \\
\hline NGC 2841 & 092202.67 & +505835.7 & $1.11 \pm 0.03$ & $0.51 \pm 0.10$ & 0.155 \\
\hline NGC 2976 Enuc. $1 \mathrm{~A}$ & 094705.14 & +675551.4 & $0.75 \pm 0.03$ & $1.08 \pm 0.16$ & 1.425 \\
\hline NGC 2976 Enuc. $1 \mathrm{~B}$ & 094707.53 & +675554.4 & $1.58 \pm 0.05$ & $5.40 \pm 0.81$ & 1.205 \\
\hline NGC 2976 Enuc. $1 \mathrm{C}$ & 094707.85 & +675548.4 & $0.26 \pm 0.02$ & $0.86 \pm 0.13$ & 1.114 \\
\hline NGC 2976 Enuc. 1 D & 094708.28 & +675553.2 & $0.42 \pm 0.02$ & $2.54 \pm 0.38$ & 1.141 \\
\hline NGC 2976 & 094713.54 & +675455.8 & $0.23 \pm 0.02$ & $5.07 \pm 0.76$ & 0.412 \\
\hline NGC 2976 Enuc. 2 A & 094723.04 & +675405.4 & $0.15 \pm 0.02$ & $0.93 \pm 0.14$ & 1.205 \\
\hline NGC 2976 Enuc. 2 B & 094723.52 & +675355.5 & $0.50 \pm 0.03$ & $1.59 \pm 0.24$ & 1.368 \\
\hline NGC 2976 Enuc. 2 C & 094723.89 & +675403.9 & $0.09 \pm 0.02$ & $0.24 \pm 0.04$ & 1.287 \\
\hline NGC 2976 Enuc. 2 E & 094724.37 & +675403.3 & $0.14 \pm 0.02$ & $0.57 \pm 0.09$ & 1.335 \\
\hline NGC 2976 Enuc. 2 D & 094724.37 & +675354.9 & $0.58 \pm 0.03$ & $2.92 \pm 0.44$ & 1.426 \\
\hline NGC 3049 & 095449.56 & +091616.1 & $1.18 \pm 0.04$ & $3.44 \pm 0.69$ & 0.103 \\
\hline NGC 3077 A & 100318.82 & +684357.8 & $4.44 \pm 0.14$ & $7.85 \pm 0.79$ & 0.086 \\
\hline NGC 3077 B & 100319.82 & +684403.5 & $1.41 \pm 0.05$ & $5.96 \pm 0.60$ & 0.080 \\
\hline NGC 3190 & 101805.64 & +214955.9 & $0.61 \pm 0.02$ & $0.38 \pm 0.08$ & 0.098 \\
\hline NGC 3184 & 101816.89 & +412527.3 & $0.31 \pm 0.02$ & $0.60 \pm 0.12$ & 0.043 \\
\hline NGC 3198 & 101955.01 & +453259.3 & $0.25 \pm 0.02$ & $0.25 \pm 0.05$ & 0.161 \\
\hline IC 2574 A & 102843.77 & +682827.8 & $0.67 \pm 0.03$ & $0.18 \pm 0.04$ & 6.301 \\
\hline IC 2574 B & 102848.40 & +682803.5 & $0.54 \pm 0.02$ & $4.23 \pm 0.85$ & 5.254 \\
\hline
\end{tabular}


Table 5

(Continued)

\begin{tabular}{|c|c|c|c|c|c|}
\hline Source ID & $\begin{array}{l}\text { R.A. } \\
\text { (J2000) }\end{array}$ & $\begin{array}{l}\text { Decl. } \\
\text { (J2000) }\end{array}$ & $\begin{array}{c}S_{33 \mathrm{GHz}} \\
(\mathrm{mJy})\end{array}$ & $\begin{array}{c}f_{\mathrm{H}_{\alpha}} / 10^{-13} \\
\left(\mathrm{erg} \mathrm{s}^{-1} \mathrm{~cm}^{-2} \text { ) }\right.\end{array}$ & $\begin{array}{c}r_{\mathrm{G}} \\
(\mathrm{kpc})\end{array}$ \\
\hline NGC 3265 & 103106.77 & +284748.0 & $1.15 \pm 0.04$ & & 0.087 \\
\hline NGC 3351 A & 104357.64 & +114217.9 & $0.50 \pm 0.02$ & $2.26 \pm 0.45$ & 0.247 \\
\hline NGC $3351 \mathrm{~B}$ & 104357.66 & +114206.8 & $2.03 \pm 0.06$ & $6.20 \pm 1.24$ & 0.285 \\
\hline NGC $3351 \mathrm{C}$ & 104357.76 & +114219.4 & $1.20 \pm 0.04$ & $2.13 \pm 0.43$ & 0.294 \\
\hline NGC 3351 D & 104358.05 & +114216.7 & $0.62 \pm 0.03$ & $1.99 \pm 0.40$ & 0.351 \\
\hline NGC 3521 Enuc. 1 & 110546.86 & -000426.3 & $1.05 \pm 0.05$ & $1.50 \pm 0.30$ & 10.282 \\
\hline NGC 3521 Enuc. 3 & 110548.60 & +00 0023.5 & $<0.08$ & $1.35 \pm 0.27$ & 9.547 \\
\hline NGC 3521 Enuc. 2 & 110549.38 & -000325.2 & $<0.11$ & $4.17 \pm 0.83$ & 4.350 \\
\hline NGC 3521 & 110549.76 & -000155.2 & $<0.10$ & $9.48 \pm 1.90$ & 2.512 \\
\hline NGC 3621 & 111816.88 & -324901.2 & $0.32 \pm 0.03$ & $\ldots$ & 0.671 \\
\hline NGC 3627 & 112015.02 & +125929.7 & $2.17 \pm 0.07$ & $2.03 \pm 0.41$ & 0.032 \\
\hline NGC 3627 Enuc. 1 A & 112016.28 & +125749.2 & $1.47 \pm 0.05$ & $0.30 \pm 0.06$ & 4.699 \\
\hline NGC 3627 Enuc. 2 & 112016.38 & +125843.7 & $6.05 \pm 0.18$ & $5.64 \pm 1.13$ & 2.661 \\
\hline NGC 3627 Enuc. 1 B & 112016.47 & +125749.8 & $0.42 \pm 0.02$ & $0.84 \pm 0.17$ & 4.736 \\
\hline NGC 3773 & 113813.10 & +120644.7 & $0.93 \pm 0.03$ & $\ldots$ & 0.118 \\
\hline NGC 3938 Enuc. 1 & 115244.51 & +440718.7 & $0.57 \pm 0.03$ & $2.77 \pm 0.55$ & 4.978 \\
\hline NGC 3938 & 115248.83 & +440717.6 & $0.06 \pm 0.02$ & $2.47 \pm 0.49$ & 0.637 \\
\hline NGC 3938 Enuc. 2 A & 115259.94 & +440800.0 & $0.22 \pm 0.02$ & $0.63 \pm 0.13$ & 11.053 \\
\hline NGC 3938 Enuc. 2 B & 115300.22 & +440748.3 & $0.26 \pm 0.02$ & $0.73 \pm 0.15$ & 11.077 \\
\hline NGC 4254 Enuc. 2 A & 121845.22 & +142437.4 & $<0.03$ & $0.89 \pm 0.18$ & 5.111 \\
\hline NGC 4254 Enuc. 2 B & 121846.15 & +142419.1 & $0.60 \pm 0.02$ & $1.98 \pm 0.40$ & 4.667 \\
\hline NGC 4254 Enuc. $1 \mathrm{~A}$ & 121849.20 & +142357.6 & $0.29 \pm 0.02$ & $0.70 \pm 0.14$ & 4.449 \\
\hline NGC 4254 Enuc. 1 B & 121850.07 & +142406.0 & $0.34 \pm 0.02$ & $1.11 \pm 0.22$ & 3.966 \\
\hline NGC 4254 & 121850.16 & +142512.2 & $0.34 \pm 0.02$ & $6.05 \pm 1.21$ & 1.031 \\
\hline NGC 4254 Enuc. $1 \mathrm{C}$ & 121850.19 & +142418.0 & $0.44 \pm 0.02$ & $1.44 \pm 0.29$ & 3.158 \\
\hline NGC 4321 Enuc. 2 & 122249.92 & +155028.4 & $0.18 \pm 0.01$ & $1.16 \pm 0.23$ & 7.991 \\
\hline NGC $4321 \mathrm{~A}$ & 122254.63 & +154913.8 & $1.46 \pm 0.05$ & $2.75 \pm 0.55$ & 0.568 \\
\hline NGC $4321 \mathrm{~B}$ & 122254.94 & +154920.1 & $0.49 \pm 0.02$ & $0.82 \pm 0.16$ & 0.086 \\
\hline NGC $4321 \mathrm{C}$ & 122255.32 & +154915.9 & $0.62 \pm 0.03$ & $0.75 \pm 0.15$ & 0.641 \\
\hline NGC $4321 \mathrm{D}$ & 122255.34 & +154923.4 & $0.23 \pm 0.02$ & $0.11 \pm 0.02$ & 0.503 \\
\hline NGC $4321 \mathrm{E}$ & 122255.38 & +154920.7 & $0.31 \pm 0.02$ & $0.28 \pm 0.06$ & 0.547 \\
\hline NGC 4321 Enuc. 1 & 122258.53 & +154918.5 & $0.21 \pm 0.01$ & $1.96 \pm 0.39$ & 4.155 \\
\hline NGC 4536 A & 123426.92 & +0211 19.7 & $9.28 \pm 0.28$ & $4.41 \pm 0.88$ & 0.273 \\
\hline NGC $4536 \mathrm{~B}$ & 123427.30 & +021115.8 & $7.33 \pm 0.22$ & $2.38 \pm 0.48$ & 0.277 \\
\hline NGC 4559 A & 123556.18 & +275740.5 & $0.24 \pm 0.01$ & $3.22 \pm 0.64$ & 1.395 \\
\hline NGC $4559 \mathrm{~B}$ & 123556.39 & +2757 19.5 & $0.12 \pm 0.01$ & $2.99 \pm 0.60$ & 2.028 \\
\hline NGC $4559 \mathrm{C}$ & 123558.45 & +275727.9 & $0.33 \pm 0.01$ & $3.60 \pm 0.72$ & 0.563 \\
\hline NGC 4569 & 123649.80 & +130946.6 & $1.60 \pm 0.05$ & $9.57 \pm 1.91$ & 0.037 \\
\hline NGC 4579 & 123743.52 & +114905.6 & $61.29 \pm 1.84$ & $4.56 \pm 0.91$ & 0.105 \\
\hline NGC 4594 & 123959.42 & -113723.0 & $73.71 \pm 2.21$ & $1.85 \pm 0.37$ & 0.052 \\
\hline NGC 4631 Enuc. 1 & 124140.73 & +323149.1 & $0.78 \pm 0.03$ & $6.78 \pm 1.36$ & 13.672 \\
\hline NGC 4625 & 124150.72 & +411620.4 & $0.18 \pm 0.01$ & $3.32 \pm 0.50$ & 1.078 \\
\hline NGC 4631 A & 124203.34 & +323217.5 & $0.47 \pm 0.02$ & $0.17 \pm 0.03$ & 3.135 \\
\hline NGC 4631 B & 124203.58 & +323216.3 & $1.02 \pm 0.03$ & $0.29 \pm 0.06$ & 3.384 \\
\hline NGC $4631 \mathrm{C}$ & 124204.14 & +323219.0 & $0.62 \pm 0.02$ & $0.49 \pm 0.10$ & 2.701 \\
\hline NGC $4631 \mathrm{D}$ & 124204.19 & +323214.5 & $0.27 \pm 0.02$ & $0.26 \pm 0.05$ & 3.823 \\
\hline NGC $4631 \mathrm{E}$ & 124204.26 & +323225.3 & $3.68 \pm 0.11$ & $1.06 \pm 0.21$ & 1.755 \\
\hline NGC $4631 \mathrm{~F}$ & 124205.05 & +323214.5 & $0.13 \pm 0.02$ & $0.09 \pm 0.02$ & 3.863 \\
\hline NGC $4631 \mathrm{G}$ & 124205.07 & +323210.3 & $0.50 \pm 0.02$ & $0.81 \pm 0.16$ & 5.072 \\
\hline NGC $4631 \mathrm{H}$ & 124205.59 & +323230.1 & $1.31 \pm 0.04$ & $1.16 \pm 0.23$ & 1.485 \\
\hline NGC 4631 I & 124206.23 & +323231.6 & $0.98 \pm 0.03$ & $0.65 \pm 0.13$ & 1.501 \\
\hline NGC $4631 \mathrm{~K}$ & 124207.18 & +323234.3 & $0.85 \pm 0.03$ & $0.43 \pm 0.09$ & 1.858 \\
\hline NGC $4631 \mathrm{~J}$ & 124207.25 & +323229.5 & $0.47 \pm 0.02$ & $0.18 \pm 0.04$ & 0.494 \\
\hline NGC 4631L & 124207.68 & +323229.8 & $0.60 \pm 0.02$ & $0.26 \pm 0.05$ & 0.359 \\
\hline NGC $4631 \mathrm{M}$ & 124207.84 & +323234.9 & $2.09 \pm 0.06$ & $0.45 \pm 0.09$ & 1.826 \\
\hline NGC 4631N & 124208.18 & +323236.1 & $1.34 \pm 0.04$ & $0.30 \pm 0.06$ & 2.102 \\
\hline NGC 4631 Enuc. 2 A & 124221.44 & +323306.6 & $0.43 \pm 0.02$ & $1.80 \pm 0.36$ & 10.046 \\
\hline NGC 4631 Enuc. 2 B & 124221.92 & +323244.4 & $1.42 \pm 0.04$ & $6.93 \pm 1.39$ & 6.592 \\
\hline NGC $4725 \mathrm{~A}$ & 125026.56 & +253002.7 & $0.29 \pm 0.01$ & $<0.02$ & 0.043 \\
\hline NGC $4725 \mathrm{~B}$ & 125028.48 & +253022.5 & $0.36 \pm 0.02$ & $0.04 \pm 0.01$ & 1.935 \\
\hline NGC 4736 & 125053.03 & +410713.1 & $2.24 \pm 0.07$ & $2.72 \pm 0.54$ & 0.019 \\
\hline NGC 4736 Enuc. $1 \mathrm{~A}$ & 125056.33 & +410714.0 & $0.63 \pm 0.03$ & $1.61 \pm 0.32$ & 0.842 \\
\hline NGC 4736 Enuc. $1 \mathrm{~B}$ & 125056.65 & +410704.7 & $1.04 \pm 0.04$ & $2.12 \pm 0.42$ & 0.926 \\
\hline
\end{tabular}


Table 5

(Continued)

\begin{tabular}{|c|c|c|c|c|c|}
\hline Source ID & $\begin{array}{l}\text { R.A. } \\
\text { (J2000) }\end{array}$ & $\begin{array}{l}\text { Decl. } \\
\text { (J2000) }\end{array}$ & $\begin{array}{c}S_{33 \mathrm{GHz}} \\
(\mathrm{mJy})\end{array}$ & $\begin{array}{c}f_{\mathrm{H}_{\alpha}} / 10^{-13} \\
\left(\mathrm{erg} \mathrm{s}^{-1} \mathrm{~cm}^{-2}\right)\end{array}$ & $\begin{array}{c}r_{\mathrm{G}} \\
(\mathrm{kpc})\end{array}$ \\
\hline NGC 4736 Enuc. $1 \mathrm{C}$ & 125056.86 & +410647.3 & $0.61 \pm 0.03$ & $1.51 \pm 0.30$ & 1.144 \\
\hline NGC 4826 A & 125643.10 & +214054.6 & $0.15 \pm 0.01$ & $0.57 \pm 0.06$ & 0.438 \\
\hline NGC 4826 B & 125643.41 & +214100.9 & $0.74 \pm 0.03$ & $2.10 \pm 0.21$ & 0.114 \\
\hline NGC 4826 D & 125643.62 & +214059.1 & $0.92 \pm 0.03$ & $4.96 \pm 0.50$ & 0.066 \\
\hline NGC 4826 C & 125643.64 & +214103.6 & $0.63 \pm 0.02$ & $1.03 \pm 0.10$ & 0.156 \\
\hline NGC $4826 \mathrm{E}$ & 125643.86 & +214057.3 & $0.34 \pm 0.02$ & $1.08 \pm 0.11$ & 0.110 \\
\hline NGC $4826 \mathrm{~F}$ & 125644.29 & +214055.5 & $0.34 \pm 0.02$ & $0.69 \pm 0.07$ & 0.240 \\
\hline NGC 5055 & 131549.36 & +420140.3 & $0.64 \pm 0.03$ & $4.90 \pm 0.83$ & 0.309 \\
\hline NGC 5055 Enuc. $1 \mathrm{~A}$ & 131558.24 & +420025.9 & $0.24 \pm 0.02$ & $1.05 \pm 0.18$ & 5.686 \\
\hline NGC 5055 Enuc. $1 \mathrm{~B}$ & 131558.40 & +420029.5 & $0.20 \pm 0.02$ & $0.88 \pm 0.15$ & 5.549 \\
\hline NGC 5194 Enuc. 6 A & 132939.35 & +470840.7 & $0.45 \pm 0.02$ & $1.06 \pm 0.21$ & 12.306 \\
\hline NGC 5194 Enuc. 6 B & 132939.35 & +470836.2 & $0.19 \pm 0.01$ & $0.40 \pm 0.08$ & 12.445 \\
\hline NGC 5194 Enuc. 3 A & 132943.69 & +471000.7 & $0.14 \pm 0.01$ & $0.41 \pm 0.08$ & 7.689 \\
\hline NGC 5194 Enuc. 2 A & 132944.07 & +471022.8 & $1.11 \pm 0.04$ & $5.10 \pm 1.02$ & 6.866 \\
\hline NGC 5194 Enuc. 3 B & 132944.54 & +470959.2 & $0.17 \pm 0.01$ & $1.10 \pm 0.22$ & 7.291 \\
\hline NGC 5194 Enuc. 3 C & 132945.16 & +470956.8 & $0.45 \pm 0.02$ & $0.95 \pm 0.19$ & 7.052 \\
\hline NGC 5194 Enuc. 11 A & 132947.05 & +471340.7 & $0.23 \pm 0.01$ & $1.12 \pm 0.22$ & 4.946 \\
\hline NGC 5194 Enuc. 11 B & 132947.55 & +471325.1 & $0.21 \pm 0.01$ & $0.36 \pm 0.07$ & 4.357 \\
\hline NGC 5194 Enuc. 1 A & 132949.42 & +47 1240.6 & $0.99 \pm 0.03$ & $1.53 \pm 0.31$ & 2.570 \\
\hline NGC 5194 Enuc. 11 D & 132949.55 & +471327.8 & $0.16 \pm 0.01$ & $0.28 \pm 0.06$ & 4.049 \\
\hline NGC 5194 Enuc. $11 \mathrm{C}$ & 132949.55 & +471359.9 & $0.12 \pm 0.01$ & $0.03 \pm 0.01$ & 5.215 \\
\hline NGC 5194 A & 132949.93 & +471131.0 & $0.30 \pm 0.02$ & $0.54 \pm 0.11$ & 1.875 \\
\hline NGC 5194 Enuc. $11 \mathrm{E}$ & 132950.47 & +471344.9 & $0.16 \pm 0.01$ & $0.39 \pm 0.08$ & 4.631 \\
\hline NGC 5194 Enuc. $11 \mathrm{~F}$ & 132950.94 & +471343.7 & $0.11 \pm 0.01$ & $0.10 \pm 0.02$ & 4.595 \\
\hline NGC 5194 B & 132951.55 & +471208.2 & $0.34 \pm 0.02$ & $0.98 \pm 0.20$ & 1.043 \\
\hline NGC 5194 Enuc. 1 B & 132952.04 & +471243.3 & $0.63 \pm 0.02$ & $2.83 \pm 0.57$ & 2.308 \\
\hline NGC 5194 C & 132952.58 & +471153.5 & $1.72 \pm 0.05$ & $3.97 \pm 0.79$ & 0.402 \\
\hline NGC 5194 D & 132952.70 & +471142.7 & $0.57 \pm 0.02$ & $1.66 \pm 0.33$ & 0.012 \\
\hline NGC 5194 E & 132952.76 & +471139.1 & $0.56 \pm 0.02$ & $1.31 \pm 0.26$ & 0.148 \\
\hline NGC 5194 Enuc. $1 \mathrm{C}$ & 132953.19 & +47 1239.7 & $0.30 \pm 0.02$ & $1.73 \pm 0.35$ & 2.348 \\
\hline NGC 5194 Enuc. 4 & 132955.49 & +471401.9 & $0.51 \pm 0.02$ & $0.13 \pm 0.03$ & 6.205 \\
\hline NGC $5194 \mathrm{~F}$ & 132955.82 & +471144.8 & $0.96 \pm 0.03$ & $1.95 \pm 0.39$ & 1.920 \\
\hline NGC 5194 Enuc. 10 & 132956.52 & +471046.0 & $0.85 \pm 0.03$ & $2.65 \pm 0.53$ & 2.743 \\
\hline NGC 5194 Enuc. 5 & 132959.63 & +471359.8 & $0.51 \pm 0.02$ & $0.10 \pm 0.02$ & 7.755 \\
\hline NGC 5194 Enuc. 9 & 132959.84 & +471112.6 & $0.49 \pm 0.02$ & $1.22 \pm 0.24$ & 4.166 \\
\hline NGC 5194 Enuc. 7 A & 133000.91 & +470929.5 & $0.31 \pm 0.02$ & $1.03 \pm 0.21$ & 6.159 \\
\hline NGC 5194 Enuc. 8 & 133001.51 & +471251.4 & $0.66 \pm 0.03$ & $1.45 \pm 0.29$ & 6.659 \\
\hline NGC 5194 Enuc. 7 B & 133002.38 & +470949.0 & $0.41 \pm 0.02$ & $2.39 \pm 0.48$ & 6.323 \\
\hline NGC 5194 Enuc. 7 C & 133002.77 & +470957.1 & $0.16 \pm 0.02$ & $1.16 \pm 0.23$ & 6.364 \\
\hline NGC 5194 Enuc. 7 D & 133003.47 & +470940.9 & $0.09 \pm 0.02$ & $0.78 \pm 0.16$ & 6.952 \\
\hline NGC 5398 & 140120.13 & -330410.8 & $1.77 \pm 0.06$ & $\ldots$ & 1.406 \\
\hline NGC 5457 Enuc. $6 \mathrm{~A}$ & 140228.24 & +54 1626.3 & $1.32 \pm 0.04$ & $7.80 \pm 1.56$ & 15.713 \\
\hline NGC 5457 Enuc. 6 B & 140229.64 & +541615.5 & $1.08 \pm 0.04$ & $3.33 \pm 0.67$ & 15.547 \\
\hline NGC 5457 Enuc. 6 C & 140230.60 & +541609.8 & $0.58 \pm 0.02$ & $2.80 \pm 0.56$ & 15.414 \\
\hline NGC 5457 Enuc. 6 D & 140230.63 & +541601.1 & $0.30 \pm 0.02$ & $1.16 \pm 0.23$ & 15.567 \\
\hline NGC 5457 Enuc. 2 & 140255.10 & +542227.8 & $0.46 \pm 0.02$ & $2.61 \pm 0.52$ & 6.428 \\
\hline NGC 5457 Enuc. 5 & 140301.17 & +541429.3 & $2.13 \pm 0.07$ & $10.36 \pm 2.07$ & 13.102 \\
\hline NGC 5457 Enuc. $1 \mathrm{~A}$ & 140311.16 & +542100.8 & $0.13 \pm 0.01$ & $0.77 \pm 0.15$ & 0.473 \\
\hline NGC 5457 & 140312.43 & +542054.6 & $0.38 \pm 0.02$ & $1.62 \pm 0.32$ & 0.092 \\
\hline NGC 5457 Enuc. 1 B & 140312.53 & +542058.1 & $0.42 \pm 0.02$ & $1.69 \pm 0.34$ & 0.044 \\
\hline NGC 5457 Enuc. 3 A & 140338.32 & +541849.9 & $0.15 \pm 0.02$ & $1.04 \pm 0.21$ & 9.334 \\
\hline NGC 5457 Enuc. 3 B & 140339.86 & +541856.8 & $0.75 \pm 0.03$ & $2.67 \pm 0.53$ & 9.645 \\
\hline NGC 5457 Enuc. 3 C & 140341.40 & +541904.3 & $7.11 \pm 0.21$ & $17.84 \pm 3.57$ & 9.964 \\
\hline NGC 5457 Enuc. 3 D & 140342.98 & +54 1924.7 & $0.47 \pm 0.03$ & $0.54 \pm 0.11$ & 10.133 \\
\hline NGC 5457 Enuc. 4 A & 140351.90 & +542152.5 & $0.13 \pm 0.01$ & $0.75 \pm 0.15$ & 12.107 \\
\hline NGC 5457 Enuc. 4 B & 140353.07 & +542156.1 & $0.16 \pm 0.01$ & $0.60 \pm 0.12$ & 12.471 \\
\hline NGC 5457 Enuc. $4 \mathrm{C}$ & 140353.13 & +542206.6 & $0.55 \pm 0.02$ & $2.40 \pm 0.48$ & 12.520 \\
\hline NGC 5457 Enuc. 4 D & 140353.99 & +542211.4 & $0.43 \pm 0.02$ & $1.83 \pm 0.37$ & 12.797 \\
\hline NGC 5457 Enuc. 7 A & 140428.61 & +542352.7 & $0.83 \pm 0.03$ & $4.66 \pm 0.93$ & 23.671 \\
\hline NGC 5457 Enuc. 7 B & 140429.23 & +542353.0 & $0.77 \pm 0.03$ & $4.10 \pm 0.82$ & 23.857 \\
\hline NGC 5457 Enuc. 7 C & 140429.47 & +542347.3 & $2.28 \pm 0.07$ & $4.87 \pm 0.97$ & 23.901 \\
\hline NGC 5474 & 140500.29 & +533956.3 & $0.03 \pm 0.01$ & $2.73 \pm 0.55$ & 0.540 \\
\hline NGC 5713 Enuc. 2 A & 144010.56 & -001747.6 & $0.12 \pm 0.01$ & $0.13 \pm 0.03$ & 3.251 \\
\hline
\end{tabular}


Table 5

(Continued)

\begin{tabular}{|c|c|c|c|c|c|}
\hline Source ID & $\begin{array}{l}\text { R.A. } \\
\text { (J2000) }\end{array}$ & $\begin{array}{l}\text { Decl. } \\
(\mathrm{J} 2000)\end{array}$ & $\begin{array}{l}S_{33 \mathrm{GHz}} \\
(\mathrm{mJy})\end{array}$ & $\begin{array}{c}f_{\mathrm{H}_{\alpha}} / 10^{-13} \\
\left(\mathrm{erg} \mathrm{s}^{-1} \mathrm{~cm}^{-2}\right)\end{array}$ & $\begin{array}{c}r_{\mathrm{G}} \\
(\mathrm{kpc})\end{array}$ \\
\hline NGC 5713 A & $14 \quad 4010.74$ & -00 1719.8 & $1.27 \pm 0.04$ & $0.49 \pm 0.10$ & 1.324 \\
\hline NGC 5713 Enuc. 2 B & 144010.74 & -001738.0 & $0.21 \pm 0.02$ & $0.43 \pm 0.09$ & 2.252 \\
\hline NGC 5713 Enuc. 2 C & 144010.88 & -001752.1 & $0.19 \pm 0.02$ & $0.36 \pm 0.07$ & 3.473 \\
\hline NGC 5713 Enuc. 2 D & 144010.94 & -001736.2 & $0.26 \pm 0.02$ & $0.43 \pm 0.09$ & 1.915 \\
\hline NGC 5713 B & 144011.06 & -00 1719.2 & $1.29 \pm 0.04$ & $0.93 \pm 0.19$ & 0.774 \\
\hline NGC 5713 C & 144011.40 & -00 1719.5 & $2.75 \pm 0.08$ & $1.53 \pm 0.31$ & 0.184 \\
\hline NGC 5713 Enuc. 1 & 144012.08 & -001750.5 & $<0.03$ & $0.43 \pm 0.09$ & 3.371 \\
\hline NGC 5866 & 150629.50 & +554547.4 & $4.09 \pm 0.12$ & $\ldots$ & 0.102 \\
\hline NGC 6946 Enuc. 4 A & 203419.16 & +601003.9 & $0.69 \pm 0.02$ & $\ldots$ & 9.184 \\
\hline NGC 6946 Enuc. 4 B & 203419.88 & +601006.6 & $1.56 \pm 0.05$ & $\ldots$ & 9.033 \\
\hline NGC 6946 Enuc. 4 C & 203421.41 & +601018.0 & $0.69 \pm 0.02$ & $\ldots$ & 8.806 \\
\hline NGC 6946 Enuc. 4 D & 203422.70 & +601033.9 & $1.33 \pm 0.04$ & $\ldots$ & 8.743 \\
\hline NGC 6946 Enuc. 8 & 203432.28 & +601019.0 & $1.51 \pm 0.05$ & $5.98 \pm 1.20$ & 6.116 \\
\hline NGC 6946 Enuc. 5 & 203439.36 & +600453.3 & $0.48 \pm 0.02$ & $7.01 \pm 1.40$ & 9.673 \\
\hline NGC 6946 Enuc. 3 A & 203449.83 & +601240.4 & $0.24 \pm 0.01$ & $2.45 \pm 0.49$ & 7.734 \\
\hline NGC 6946 Enuc. 3 B & 203451.19 & +601242.2 & $0.20 \pm 0.01$ & $2.31 \pm 0.46$ & 7.722 \\
\hline NGC 6946 A & 203451.30 & +600938.6 & $1.39 \pm 0.05$ & $4.20 \pm 0.84$ & 0.983 \\
\hline NGC 6946 Enuc. 3 C & 203452.20 & +601243.7 & $0.47 \pm 0.02$ & $7.15 \pm 1.43$ & 7.730 \\
\hline NGC 6946 B & 203452.26 & +600914.3 & $14.17 \pm 0.43$ & $15.37 \pm 3.07$ & 0.016 \\
\hline NGC $6946 \mathrm{C}$ & 203452.82 & +600930.2 & $0.52 \pm 0.03$ & $1.60 \pm 0.32$ & 0.591 \\
\hline NGC 6946 Enuc. 6 A & 203505.24 & +601056.4 & $0.13 \pm 0.02$ & $2.22 \pm 0.44$ & 4.666 \\
\hline NGC 6946 Enuc. 6 B & 203506.04 & +601100.0 & $0.51 \pm 0.02$ & $2.45 \pm 0.49$ & 4.886 \\
\hline NGC 6946 Enuc. 6 C & 203506.20 & +601056.4 & $0.35 \pm 0.02$ & $1.66 \pm 0.33$ & 4.823 \\
\hline NGC 6946 Enuc. 6 D & 203506.56 & +601059.1 & $0.11 \pm 0.02$ & $0.91 \pm 0.18$ & 4.949 \\
\hline NGC 6946 Enuc. 6 E & 203507.05 & +601045.3 & $0.20 \pm 0.02$ & $1.21 \pm 0.24$ & 4.717 \\
\hline NGC 6946 Enuc. 9 & 203511.09 & +600857.5 & $1.48 \pm 0.05$ & $8.40 \pm 1.68$ & 5.071 \\
\hline NGC 6946 Enuc. 7 A & 203512.93 & +600850.2 & $0.81 \pm 0.03$ & $3.27 \pm 0.65$ & 5.630 \\
\hline NGC 6946 Enuc. 7 B & 203514.10 & +600851.7 & $0.13 \pm 0.02$ & $0.74 \pm 0.15$ & 5.916 \\
\hline NGC 6946 Enuc. 1 & 203516.76 & +601100.3 & $0.81 \pm 0.03$ & $7.72 \pm 1.54$ & 6.985 \\
\hline NGC 6946 Enuc. 2 A & 203523.61 & +600948.9 & $0.36 \pm 0.02$ & $5.20 \pm 1.04$ & 8.131 \\
\hline NGC 6946 Enuc. 2 B & 203525.50 & +600958.8 & $1.96 \pm 0.06$ & $25.89 \pm 5.18$ & 8.626 \\
\hline NGC 7331 & 223702.96 & +342506.5 & $0.15 \pm 0.04$ & $6.15 \pm 1.23$ & 2.930 \\
\hline NGC 7793 Enuc. 3 & 235748.87 & -323452.8 & $<0.12$ & $5.65 \pm 1.13$ & 0.992 \\
\hline NGC 7793 Enuc. 1 & 235749.44 & -323712.4 & $0.30 \pm 0.03$ & $7.08 \pm 1.42$ & 2.953 \\
\hline NGC 7793 & 235749.56 & -323525.2 & $<0.07$ & $3.63 \pm 0.73$ & 0.093 \\
\hline NGC 7793 Enuc. 2 & 235755.74 & -323522.3 & $<0.07$ & $5.67 \pm 1.13$ & 1.468 \\
\hline
\end{tabular}

(This table is available in machine-readable form.)

of Aniano et al. (2011), we convolved the $24 \mu \mathrm{m}$ maps with a kernel that both subtracts out the complex $24 \mu \mathrm{m}$ PSF and restores the image with a Gaussian PSF having an FWHM of $7^{\prime \prime}$.

Using the resolution-matched images, we measured the flux density within apertures having a $7^{\prime \prime}$ diameter. No attempt was made to apply an aperture correction to the convolved-map photometry, as we are only interested in using these data to compare relative values measured at these three bands. The majority of these 179 apertures were created by centering a $7^{\prime \prime}$ diameter circle around the peak pixel in each native resolution aperture and removing apertures that overlap significantly with others or are strongly contaminated by emission from nearby bright sources. Additionally, we have created $7^{\prime \prime}$ apertures for 17 "diffuse detections" where the $33 \mathrm{GHz}$ emission is intrinsically faint and diffuse such that it falls below our compact source detection threshold on $2^{\prime \prime}$ scales, but constitutes a $>5 \sigma$ detection on $7^{\prime \prime}$ scales.

The corresponding $33 \mathrm{GHz}$ flux densities, $\mathrm{H} \alpha$ line fluxes, and $24 \mu \mathrm{m}$ flux densities for each region detected with an $\mathrm{S} / \mathrm{N}>3$ are given in Table 6 . Similar to the naming convention for the photometry carried out at the full resolution of the
$33 \mathrm{GHz}$ maps, sources are named according to the nearest $33 \mathrm{GHz}$ image, with an alphabetical suffix if there are multiple regions corresponding to one image. However, we distinguish individual sources identified in the smoothed maps by instead using a lowercase letter. For example, "NGC 2403 Enuc 2. b" is 1 of 2 regions in the image of extranuclear region 2 in NGC 2403, and is composed of the sum contribution of NGC 2403 Enuc 2. B and NGC 2403 Enuc 2. C in the fullresolution maps. For sources that are not detected at this significance we provide a corresponding $3 \sigma$ upper limit. As done for sources listed in Table 5, we similarly provide a measure of the galactocentric radius in units of kiloparsecs for each position.

\section{Results}

\subsection{Comparison with Single-dish}

As a first test to see how much emission might be resolved out of these snapshot-like $33 \mathrm{GHz}$ images, we perform a comparison between the emission recovered in the interferometric images with the photometry obtained with the GBT given in Murphy et al. (2012). To do this, we multiply the VLA 

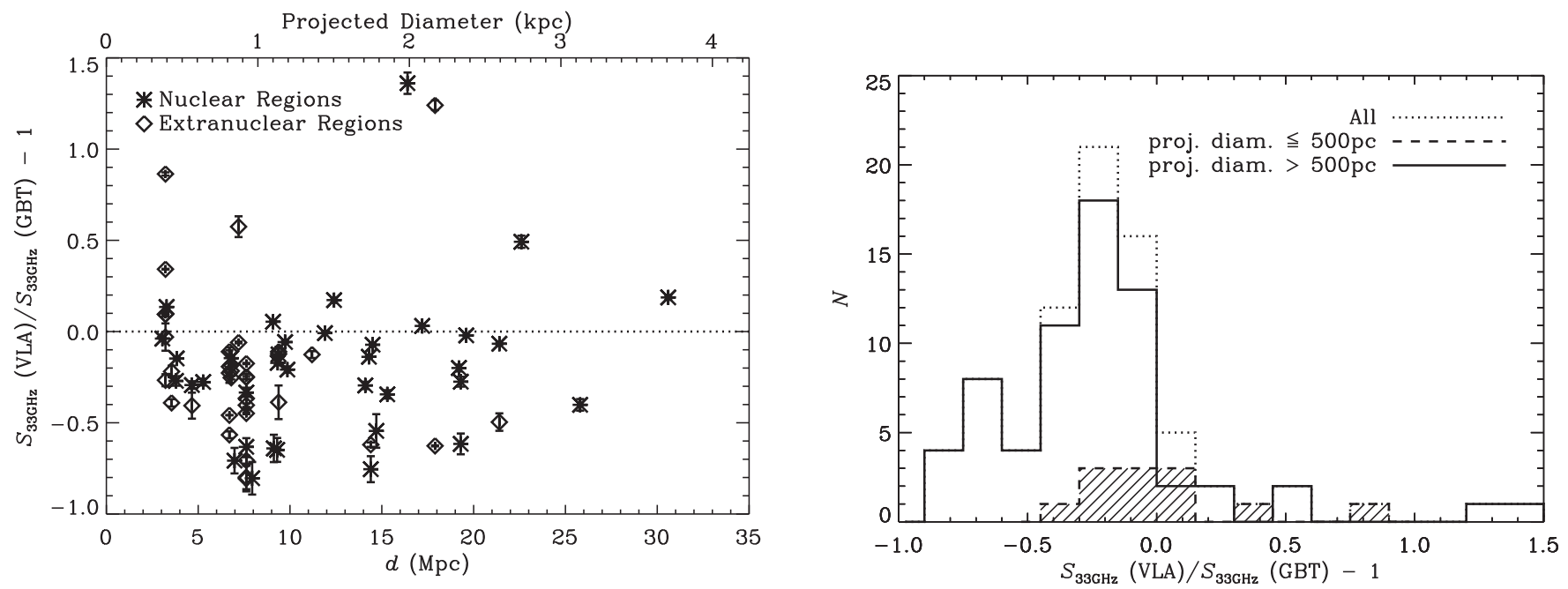

Figure 2. Left: the relative difference between the VLA and GBT measured $33 \mathrm{GHz}$ flux densities plotted against distance for sources detected at the $5 \sigma$ significance level in both data sets. The upper abscissa identifies the size of the projected diameter of the $25^{\prime \prime}$ GBT beam. For the VLA, flux densities were measured by multiplying the VLA image by an elliptical Gaussian to simulate the GBT observations (see Section 3.1). NGC 4579, which hosts an AGN, is the data point for which the 33 GHz VLA flux density is more than a factor of 2 larger than the corresponding $33 \mathrm{GHz}$ GBT flux density. Right: histogram of the relative difference between the VLA and GBT $33 \mathrm{GHz}$ flux densities for sources detected at the $5 \sigma$ significance level in both data sets using bins of 0.15 (dotted line). Individual histograms of those sources for which the projected diameter of the $25^{\prime \prime} \mathrm{GBT}$ beam is larger (solid line) or smaller (dashed line/hatch filled) than $\approx 500$ pc are also shown. What is clearly evident is that the VLA flux densities are systematically lower than what was recovered by the GBT. The median $33 \mathrm{GHz}$ VLA-to-GBT flux density ratio is $0.78 \pm 0.04$, with median absolute deviation of 0.27 . For the 12 sources in which the $25^{\prime \prime}$ GBT beam projects to a linear diameter of $\lesssim 500$ pc, the median 33 GHz VLA-to-GBT flux density ratio is $0.97 \pm 0.10$, with a median absolute deviation of 0.28 , suggesting that this difference between the GBT and VLA flux densities likely arises from diffuse non-thermal synchrotron emission associated with CR electrons as they propagate away from their birth sites in supernova remnants near $\mathrm{H}$ II regions.

image by an elliptical Gaussian of peak unity, having major/ minor axes and position angles based on the interferometric synthesized beams such that the convolution of the two results in a circular Gaussian beam with a FWHM of $25^{\prime \prime}$, to match the typical beam size of the GBT at $33 \mathrm{GHz}$. Since we assume a perfect Gaussian and do not account for additional emission arising from sidelobes in the actual GBT beam, these simulated observations will in most cases only provide a lower limit compared to what was measured by the GBT. However, we assume that this is likely a small (few percent) effect given that the sidelobes from the GBT measurements, when measurable, had an amplitude that is $2 \%$ of the beam peak, on average (Murphy et al. 2012).

We compare these measured flux densities against what was measured by the GBT as a function of galaxy distance in the left panel of Figure 2 for sources detected at $>5 \sigma$ in both data sets. In the right panel of Figure 2, we plot the histogram of these sources using bins of 0.15 and highlight sources for which the $25^{\prime \prime}$ GBT beam projects to a linear diameter of $\lesssim 500$ pc. What we find is that the VLA is typically missing $\approx 20 \%$ of the total flux density recovered by the GBT. The median $33 \mathrm{GHz}$ VLA-to-GBT flux density ratio is $0.78 \pm 0.04$, with a median absolute deviation of 0.27 . The most likely reason for this discrepancy between the VLA and GBT photometry is that the GBT beam is picking up diffuse emission extended on scales greater than the largest angular scale that these VLA $33 \mathrm{GHz}$ data are sensitive to (i.e., $\gtrsim 24^{\prime \prime}$ ). However, we do not expect this to affect our aperture photometry results since we are only integrating on selected bright regions on the scale of a few arcseconds, where contributions from large-scale diffuse emission on scales $\gtrsim 24$ " should be negligible.

Furthermore, on such scales the bulk of the emission being resolved out by our $33 \mathrm{GHz}$ interferometric observations is likely diffuse non-thermal synchrotron emission associated with $\mathrm{CR}$ electrons as they propagate away from their birth sites in supernova remnants near $\mathrm{H}$ II regions. For example, for the 12 sources in which the $25^{\prime \prime}$ GBT beam projects to a linear diameter of $\lesssim 500 \mathrm{pc}$, the median $33 \mathrm{GHz}$ VLA-to-GBT flux density ratio is $0.97 \pm 0.10$, with a median absolute deviation of 0.28 . Thus, given that the average thermal fraction at $33 \mathrm{GHz}$ reported by Murphy et al. (2012) was $76 \%$ for their entire sample (and $>90 \%$ on average for sources resolved on scales $\lesssim 500 \mathrm{pc}$ ), this suggests that on the $\approx 30-300 \mathrm{pc}$ scales of these VLA observations, the $33 \mathrm{GHz}$ thermal fractions are most likely $\gtrsim 90 \%$. Consequently, the $80 \%$ thermal fraction of the GBT analysis is completely consistent with the measured $33 \mathrm{GHz}$ VLA-to-GBT flux density ratio if all compact emission is powered by free-free radiation, while the non-thermal component is completely diffuse. We note that there is a minority of sources having values above unity, for which the VLA appears to be recovering more emission than the GBT. These occurrences most likely arise due to sources hosting a variable AGN (e.g., NGC 4579 for which the $33 \mathrm{GHz}$ VLA flux density is more than a factor of 2 larger than the corresponding $33 \mathrm{GHz}$ GBT flux density) or situations where the GBT reference beam used for sky subtraction by nodding $1 ! 3$ away from the source position landed on bright regions of the galaxies (e.g., NGC 3938 Enuc. 1; see Murphy et al. 2012).

\section{2. $33 \mathrm{GHz}$ and $\mathrm{H \alpha}$ Morphologies}

At the $2^{\prime \prime}(\approx 30-300 \mathrm{pc})$ scales probed by our $33 \mathrm{GHz}$ observations, we are primarily sensitive to compact emission from individual star-forming complexes and galaxy nuclei. As a visual demonstration of this, we compared the $33 \mathrm{GHz}$ morphologies of our targets with their $\mathrm{H} \alpha$ and $24 \mu \mathrm{m}$ morphologies. To investigate at the highest resolution, the $\approx 2^{\prime \prime}$ beam radio images and $\approx 1^{\prime \prime}-2^{\prime \prime}$ seeing-limited $\mathrm{H} \alpha$ images were compared at their native resolutions. To match the Spitzer PSF for the $33 \mathrm{GHz} / 24 \mu \mathrm{m}$ comparison, we smoothed the $33 \mathrm{GHz}$ images to a $7^{\prime \prime}$ circular beam with the CASA task 
Table 6

Source Photometry at 7 " Resolution

\begin{tabular}{|c|c|c|c|c|c|c|}
\hline Source ID & $\begin{array}{c}\text { R.A. } \\
(\mathrm{J} 2000)\end{array}$ & $\begin{array}{c}\text { Decl. } \\
(\mathrm{J} 2000)\end{array}$ & $\begin{array}{c}S_{33 \mathrm{GHz}} \\
(\mathrm{mJy})\end{array}$ & $\begin{array}{c}f_{\mathrm{H}_{\alpha}} / 10^{-13} \\
\left(\mathrm{erg} \mathrm{s}^{-1} \mathrm{~cm}^{-2}\right)\end{array}$ & $\begin{array}{c}f_{24 \mu \mathrm{m}} \\
(\mathrm{mJy})\end{array}$ & $\begin{array}{c}r_{\mathrm{G}} \\
(\mathrm{kpc})\end{array}$ \\
\hline NGC 0337a & 005950.02 & -073433.9 & $0.23 \pm 0.01$ & $1.50 \pm 0.30$ & $48.04 \pm 2.40$ & 0.945 \\
\hline NGC $0337 b$ & 005950.68 & -073457.6 & $0.62 \pm 0.02$ & $1.72 \pm 0.34$ & $80.48 \pm 4.02$ & 1.966 \\
\hline NGC 0337c & 005951.99 & -073454.9 & $0.10 \pm 0.01$ & $0.52 \pm 0.10$ & $8.54 \pm 0.43$ & 3.227 \\
\hline NGC 0337d & 005952.16 & -073438.2 & $0.22 \pm 0.01$ & $1.32 \pm 0.26$ & $24.25 \pm 1.21$ & 4.061 \\
\hline NGC 0628 Enuc. 4 & 013635.72 & +155007.2 & $0.12 \pm 0.01$ & $0.49 \pm 0.10$ & $10.30 \pm 0.51$ & 7.608 \\
\hline NGC 0628 Enuc. 2 & 013637.65 & +154507.2 & $0.18 \pm 0.02$ & $0.43 \pm 0.09$ & $42.11 \pm 2.11$ & 4.468 \\
\hline NGC 0628 Enuc. 3 & 013638.78 & +154423.2 & $0.19 \pm 0.03$ & $0.78 \pm 0.16$ & $31.08 \pm 1.55$ & 5.720 \\
\hline NGC 0628 & 013641.70 & +154659.0 & $<0.06$ & $0.06 \pm 0.01$ & $2.37 \pm 0.12$ & 0.071 \\
\hline NGC 0628 Enuc. 1 & 013645.27 & +154748.3 & $0.29 \pm 0.03$ & $0.71 \pm 0.14$ & $78.59 \pm 3.93$ & 2.478 \\
\hline NGC 0855 & 021403.68 & +275237.9 & $0.21 \pm 0.01$ & $\ldots$ & $19.62 \pm 0.98$ & 0.202 \\
\hline NGC 0925 & 022717.00 & +333443.0 & $<0.04$ & $0.30 \pm 0.06$ & $3.40 \pm 0.17$ & 0.183 \\
\hline NGC 1097 Enuc. 2 & 024614.40 & -301504.0 & $0.05 \pm 0.01$ & $\ldots$ & $3.51 \pm 0.18$ & 7.435 \\
\hline NGC 1097 & 024618.98 & -301628.8 & $2.30 \pm 0.08$ & $\ldots$ & $158.45 \pm 7.92$ & 0.020 \\
\hline NGC 1097 Enuc. 1 a & 024622.56 & -301729.9 & $0.08 \pm 0.01$ & $\ldots$ & $12.30 \pm 0.62$ & 5.416 \\
\hline NGC 1097 Enuc. 1 b & 024622.93 & -301748.1 & $0.12 \pm 0.01$ & $\ldots$ & $11.02 \pm 0.55$ & 6.817 \\
\hline NGC 1097 Enuc. $1 \mathrm{c}$ & 024624.06 & -301750.9 & $0.07 \pm 0.01$ & $\ldots$ & $8.52 \pm 0.43$ & 7.392 \\
\hline NGC 1266 & 031600.76 & -022537.1 & $4.58 \pm 0.15$ & $\ldots$ & $458.85 \pm 22.94$ & 0.270 \\
\hline NGC 1377 & 033638.90 & -205406.0 & $<0.08$ & $\ldots$ & $705.71 \pm 35.29$ & 0.580 \\
\hline NGC 1482 & 035438.97 & -203007.8 & $6.71 \pm 0.21$ & $\ldots$ & $1394.77 \pm 69.74$ & 0.124 \\
\hline NGC 2403 Enuc. 5 & 073619.84 & +653705.5 & $0.56 \pm 0.02$ & $2.21 \pm 0.44$ & $54.79 \pm 2.74$ & 3.464 \\
\hline NGC 2403 Enuc. 6 & 073628.69 & +653349.4 & $0.30 \pm 0.01$ & $1.14 \pm 0.23$ & $9.36 \pm 0.47$ & 5.380 \\
\hline NGC 2403 Enuc. 1 a & 073642.06 & +653651.9 & $0.13 \pm 0.01$ & $0.15 \pm 0.03$ & $3.73 \pm 0.19$ & 1.087 \\
\hline NGC 2403 Enuc. 1 b & 073645.50 & +653700.9 & $0.55 \pm 0.02$ & $2.80 \pm 0.56$ & $87.21 \pm 4.36$ & 1.192 \\
\hline NGC 2403 Enuc. 2 a & 073649.11 & +653651.7 & $0.25 \pm 0.02$ & $0.68 \pm 0.14$ & $36.22 \pm 1.81$ & 1.113 \\
\hline NGC 2403 & 073650.00 & +653604.0 & $<0.03$ & $0.16 \pm 0.03$ & $3.39 \pm 0.17$ & 0.000 \\
\hline NGC 2403 Enuc. 2 b & 073652.36 & +653646.9 & $0.49 \pm 0.02$ & $2.12 \pm 0.42$ & $61.17 \pm 3.06$ & 1.249 \\
\hline NGC 2403 Enuc. 3 & 073706.95 & +653639.0 & $1.62 \pm 0.05$ & $4.92 \pm 0.98$ & $294.85 \pm 14.74$ & 2.811 \\
\hline NGC 2403 Enuc. 4 & 073718.19 & +653348.1 & $0.33 \pm 0.01$ & $0.74 \pm 0.15$ & $17.82 \pm 0.89$ & 3.455 \\
\hline Holmberg II & 081913.06 & +704308.0 & $0.21 \pm 0.02$ & $1.49 \pm 0.30$ & $16.70 \pm 0.83$ & 0.738 \\
\hline NGC 2798 & 091722.85 & +420000.4 & $2.06 \pm 0.06$ & $4.46 \pm 0.89$ & $1132.59 \pm 56.63$ & 0.144 \\
\hline NGC 2841 & 092202.67 & +505835.7 & $0.56 \pm 0.02$ & $0.24 \pm 0.05$ & $13.12 \pm 0.66$ & 0.155 \\
\hline NGC 2976 Enuc. 1 a & 094705.19 & +675552.0 & $0.35 \pm 0.02$ & $0.58 \pm 0.12$ & $27.97 \pm 1.40$ & 1.420 \\
\hline NGC 2976 Enuc. 1 b & 094707.64 & +675554.7 & $0.86 \pm 0.03$ & $3.09 \pm 0.62$ & $133.92 \pm 6.70$ & 1.201 \\
\hline NGC 2976 & 094713.49 & +675454.0 & $0.09 \pm 0.02$ & $0.50 \pm 0.10$ & $7.49 \pm 0.37$ & 0.462 \\
\hline NGC 2976 Enuc. 2 a & 094723.83 & +675354.9 & $0.45 \pm 0.02$ & $1.90 \pm 0.38$ & $55.16 \pm 2.76$ & 1.394 \\
\hline NGC 2976 Enuc. 2 b & 094723.94 & +675402.1 & $0.21 \pm 0.02$ & $1.09 \pm 0.22$ & $23.23 \pm 1.16$ & 1.310 \\
\hline NGC 3049 & 095449.56 & +091616.1 & $0.54 \pm 0.02$ & $1.18 \pm 0.24$ & $191.34 \pm 11.56$ & 0.103 \\
\hline NGC 3190 & 101805.64 & +214955.9 & $0.26 \pm 0.02$ & $0.15 \pm 0.03$ & $31.69 \pm 1.58$ & 0.098 \\
\hline NGC 3184 & 101816.94 & +412527.0 & $0.14 \pm 0.01$ & $0.25 \pm 0.05$ & $57.85 \pm 2.89$ & 0.063 \\
\hline NGC 3198 & 101954.99 & +453259.3 & $0.12 \pm 0.02$ & $0.12 \pm 0.02$ & $184.89 \pm 9.24$ & 0.116 \\
\hline IC $2574 a$ & 102843.71 & +682826.3 & $0.28 \pm 0.02$ & $0.06 \pm 0.01$ & $5.22 \pm 0.26$ & 6.253 \\
\hline IC 2574b & 102848.40 & +682803.5 & $0.24 \pm 0.02$ & $1.74 \pm 0.35$ & $20.86 \pm 1.04$ & 5.254 \\
\hline NGC 3265 & 103106.77 & +284748.0 & $0.43 \pm 0.02$ & $\ldots$ & $147.65 \pm 7.38$ & 0.087 \\
\hline NGC 3351a & 104357.68 & +114208.0 & $0.77 \pm 0.03$ & $2.42 \pm 0.48$ & $316.70 \pm 15.83$ & 0.230 \\
\hline NGC $3351 b$ & 104357.80 & +114218.5 & $0.89 \pm 0.03$ & $2.23 \pm 0.45$ & $403.05 \pm 20.15$ & 0.258 \\
\hline NGC 3521 Enuc. 1 & 110546.30 & -000409.0 & $<0.11$ & $0.12 \pm 0.02$ & $7.32 \pm 0.37$ & 9.929 \\
\hline NGC 3521 Enuc. 3 & 110547.60 & +000033.0 & $<0.07$ & $0.10 \pm 0.02$ & $7.48 \pm 0.37$ & 9.509 \\
\hline NGC 3521 & 110548.90 & -000206.0 & $<0.05$ & $0.59 \pm 0.12$ & $14.37 \pm 0.72$ & 0.625 \\
\hline NGC 3521 Enuc. 2 a & 110549.34 & -000324.2 & $0.15 \pm 0.03$ & $0.24 \pm 0.05$ & $22.25 \pm 1.11$ & 4.302 \\
\hline NGC 3521 Enuc. 2 b & 110549.94 & -000355.9 & $0.11 \pm 0.03$ & $0.05 \pm 0.01$ & $2.97 \pm 0.15$ & 6.044 \\
\hline NGC 3621 & 111816.00 & -324842.0 & $<0.09$ & $\ldots$ & $7.86 \pm 0.39$ & 0.348 \\
\hline NGC 3627 & 112015.00 & +125929.4 & $1.07 \pm 0.04$ & $0.88 \pm 0.18$ & $257.22 \pm 12.86$ & 0.028 \\
\hline NGC 3627 Enuc. 1 & 112016.32 & +125749.2 & $0.85 \pm 0.03$ & $0.39 \pm 0.08$ & $177.00 \pm 8.85$ & 4.712 \\
\hline NGC 3627 Enuc. 2 & 112016.46 & +125843.4 & $1.93 \pm 0.06$ & $0.92 \pm 0.18$ & $496.59 \pm 24.83$ & 2.746 \\
\hline NGC 3773 & 113813.02 & +120643.8 & $0.38 \pm 0.02$ & $\ldots$ & $60.04 \pm 3.00$ & 0.023 \\
\hline NGC 3938 & 115248.19 & +440705.9 & $0.08 \pm 0.01$ & $0.07 \pm 0.01$ & $3.39 \pm 0.17$ & 1.407 \\
\hline NGC 3938 & 115249.50 & +440714.0 & $<0.04$ & $0.07 \pm 0.01$ & $5.68 \pm 0.28$ & 0.140 \\
\hline NGC 3938 Enuc. 2 a & 115300.06 & +440800.0 & $0.10 \pm 0.02$ & $0.29 \pm 0.06$ & $7.92 \pm 0.40$ & 11.158 \\
\hline NGC 3938 Enuc. 2 b & 115300.19 & +440748.3 & $0.11 \pm 0.02$ & $0.34 \pm 0.07$ & $22.10 \pm 1.10$ & 11.049 \\
\hline NGC 4254 Enuc. 2 a & 121845.78 & +142410.4 & $0.09 \pm 0.01$ & $0.36 \pm 0.07$ & $13.60 \pm 0.68$ & 5.342 \\
\hline NGC 4254 Enuc. 2 b & 121846.13 & +142418.8 & $0.12 \pm 0.01$ & $0.18 \pm 0.04$ & $11.38 \pm 0.57$ & 4.698 \\
\hline NGC 4254a & 121848.68 & +142442.5 & $0.07 \pm 0.02$ & $0.45 \pm 0.09$ & $33.20 \pm 1.66$ & 1.553 \\
\hline NGC 4254 Enuc. 1 a & 121849.20 & +142357.9 & $0.12 \pm 0.02$ & $0.23 \pm 0.05$ & $22.90 \pm 1.15$ & 4.428 \\
\hline
\end{tabular}


Table 6

(Continued)

\begin{tabular}{|c|c|c|c|c|c|c|}
\hline Source ID & $\begin{array}{c}\text { R.A. } \\
\text { (J2000) }\end{array}$ & $\begin{array}{l}\text { Decl. } \\
\text { (J2000) }\end{array}$ & $\begin{array}{c}S_{33 \mathrm{GHz}} \\
(\mathrm{mJy})\end{array}$ & $\begin{array}{c}f_{\mathrm{H}_{\alpha}} / 10^{-13} \\
\left(\mathrm{erg} \mathrm{s}^{-1} \mathrm{~cm}^{-2} \text { ) }\right.\end{array}$ & $\begin{array}{r}f_{24 \mu \mathrm{m}} \\
(\mathrm{mJy})\end{array}$ & $\begin{array}{c}r_{\mathrm{G}} \\
(\mathrm{kpc})\end{array}$ \\
\hline NGC 4254b & 121849.67 & +142459.0 & $0.11 \pm 0.02$ & $0.60 \pm 0.12$ & $54.75 \pm 2.74$ & 0.111 \\
\hline NGC 4254 Enuc. $1 \mathrm{~b}$ & 121850.01 & +142406.9 & $0.15 \pm 0.02$ & $0.49 \pm 0.10$ & $18.52 \pm 0.93$ & 3.886 \\
\hline NGC $4254 \mathrm{c}$ & 121850.10 & +142511.6 & $0.06 \pm 0.02$ & $0.37 \pm 0.07$ & $27.57 \pm 1.38$ & 0.960 \\
\hline NGC 4254 Enuc. $1 \mathrm{c}$ & 121850.19 & +142418.6 & $0.18 \pm 0.02$ & $0.46 \pm 0.09$ & $34.51 \pm 1.73$ & 3.116 \\
\hline NGC 4254d & 121851.63 & +142508.6 & $0.12 \pm 0.02$ & $0.23 \pm 0.05$ & $16.04 \pm 0.80$ & 2.344 \\
\hline NGC $4254 \mathrm{e}$ & 121851.90 & +142449.7 & $0.13 \pm 0.02$ & $0.40 \pm 0.08$ & $25.51 \pm 1.28$ & 2.813 \\
\hline NGC $4254 \mathrm{f}$ & 121851.92 & +142440.1 & $0.14 \pm 0.02$ & $0.23 \pm 0.05$ & $11.52 \pm 0.58$ & 3.149 \\
\hline NGC 4321 Enuc. 2 a & 122248.84 & +155012.8 & $0.05 \pm 0.01$ & $0.14 \pm 0.03$ & $8.59 \pm 0.43$ & 8.305 \\
\hline NGC 4321 Enuc. $2 \mathrm{~b}$ & 122249.90 & +155027.8 & $0.05 \pm 0.01$ & $0.15 \pm 0.03$ & $6.48 \pm 0.32$ & 7.979 \\
\hline NGC 4321 Enuc. 2 c & 122250.65 & +155027.2 & $0.04 \pm 0.01$ & $0.07 \pm 0.01$ & $4.81 \pm 0.24$ & 7.285 \\
\hline NGC 4321a & 122254.65 & +154919.8 & $0.45 \pm 0.02$ & $0.80 \pm 0.16$ & $89.71 \pm 4.49$ & 0.284 \\
\hline NGC $4321 b$ & 122255.13 & +154920.4 & $0.63 \pm 0.03$ & $0.89 \pm 0.18$ & $124.94 \pm 6.25$ & 0.270 \\
\hline NGC 4321 Enuc. 1 & 122258.90 & +154935.0 & $<0.03$ & $0.10 \pm 0.02$ & $4.82 \pm 0.24$ & 4.520 \\
\hline NGC 4536 & 123427.06 & +021118.2 & $4.90 \pm 0.15$ & $2.03 \pm 0.41$ & $1010.56 \pm 50.53$ & 0.126 \\
\hline NGC $4559 \mathrm{a}$ & 123556.27 & +275740.5 & $0.08 \pm 0.01$ & $0.76 \pm 0.15$ & $10.30 \pm 0.52$ & 1.304 \\
\hline NGC $4559 \mathrm{~b}$ & 123556.46 & +275721.3 & $0.05 \pm 0.01$ & $0.57 \pm 0.11$ & $6.25 \pm 0.31$ & 1.880 \\
\hline NGC $4559 \mathrm{c}$ & 123558.47 & +275729.7 & $0.11 \pm 0.01$ & $0.72 \pm 0.14$ & $18.98 \pm 0.95$ & 0.608 \\
\hline NGC 4569 & 123649.80 & +130946.6 & $0.51 \pm 0.03$ & $2.96 \pm 0.59$ & $326.78 \pm 16.34$ & 0.037 \\
\hline NGC 4579 & 123743.52 & +114905.6 & $30.13 \pm 0.91$ & $1.98 \pm 0.40$ & $101.01 \pm 5.05$ & 0.105 \\
\hline NGC 4594 & 123959.42 & -113723.0 & $36.58 \pm 1.10$ & $0.77 \pm 0.15$ & $35.64 \pm 1.78$ & 0.052 \\
\hline NGC 4631 Enuc. 1 & 124140.47 & +323149.1 & $0.14 \pm 0.01$ & $0.82 \pm 0.16$ & $7.67 \pm 0.38$ & 13.762 \\
\hline NGC 4625 & 124152.40 & +411624.0 & $<0.03$ & $0.15 \pm 0.03$ & $4.77 \pm 0.24$ & 0.139 \\
\hline NGC 4631a & 124203.43 & +323217.2 & $0.72 \pm 0.03$ & $0.35 \pm 0.07$ & $40.98 \pm 2.05$ & 3.189 \\
\hline NGC $4631 \mathrm{~b}$ & 124203.98 & +323216.0 & $0.58 \pm 0.02$ & $0.48 \pm 0.10$ & $86.71 \pm 4.34$ & 3.435 \\
\hline NGC 4631c & 124204.31 & +323225.3 & $1.29 \pm 0.04$ & $0.27 \pm 0.05$ & $171.90 \pm 8.59$ & 1.734 \\
\hline NGC 4631d & 124205.09 & +323210.6 & $0.26 \pm 0.02$ & $0.38 \pm 0.08$ & $46.49 \pm 2.32$ & 4.988 \\
\hline NGC $4631 \mathrm{e}$ & 124205.57 & +323229.5 & $0.61 \pm 0.02$ & $0.41 \pm 0.08$ & $95.52 \pm 4.78$ & 1.387 \\
\hline NGC $4631 \mathrm{f}$ & 124206.26 & +323231.9 & $0.51 \pm 0.02$ & $0.35 \pm 0.07$ & $75.60 \pm 3.78$ & 1.566 \\
\hline NGC $4631 \mathrm{~g}$ & 124207.47 & +323231.6 & $0.97 \pm 0.03$ & $0.32 \pm 0.06$ & $145.27 \pm 7.26$ & 0.960 \\
\hline NGC $4631 \mathrm{~h}$ & 124208.06 & +323234.9 & $1.29 \pm 0.04$ & $0.30 \pm 0.06$ & $276.81 \pm 13.84$ & 1.769 \\
\hline NGC 4631 Enuc. 2 a & 124221.42 & +323306.3 & $0.18 \pm 0.01$ & $0.69 \pm 0.14$ & $19.61 \pm 0.98$ & 9.974 \\
\hline NGC 4631 Enuc. $2 \mathrm{~b}$ & 124221.99 & +323245.0 & $0.36 \pm 0.02$ & $1.93 \pm 0.39$ & $42.30 \pm 2.11$ & 6.651 \\
\hline NGC 4725a & 125026.56 & +253003.0 & $0.16 \pm 0.01$ & $<0.01$ & $22.16 \pm 1.11$ & 0.044 \\
\hline NGC $4725 b$ & 125028.48 & +253021.9 & $0.19 \pm 0.01$ & $0.01 \pm 0.00$ & $0.30 \pm 0.02$ & 1.921 \\
\hline NGC 4736 & 125053.05 & +410712.8 & $1.02 \pm 0.04$ & $0.56 \pm 0.11$ & $313.33 \pm 15.67$ & 0.014 \\
\hline NGC 4736 Enuc. 1 a & 125056.41 & +4107 14.3 & $0.35 \pm 0.02$ & $0.89 \pm 0.18$ & $93.24 \pm 4.66$ & 0.864 \\
\hline NGC 4736 Enuc. $1 \mathrm{~b}$ & 125056.70 & +410705.0 & $0.39 \pm 0.02$ & $0.74 \pm 0.15$ & $91.02 \pm 4.55$ & 0.939 \\
\hline NGC 4736 Enuc. $1 \mathrm{c}$ & 125056.78 & +410647.6 & $0.26 \pm 0.02$ & $0.54 \pm 0.11$ & $93.76 \pm 4.69$ & 1.123 \\
\hline NGC 4826 & 125643.56 & +214100.6 & $1.00 \pm 0.03$ & $3.44 \pm 0.69$ & $203.63 \pm 10.18$ & 0.055 \\
\hline NGC 5055 & 131549.31 & +420145.1 & $0.12 \pm 0.02$ & $1.26 \pm 0.25$ & $41.37 \pm 2.07$ & 0.009 \\
\hline NGC 5055 Enuc. 1 & 131558.32 & +420027.4 & $0.15 \pm 0.02$ & $0.64 \pm 0.13$ & $23.38 \pm 1.17$ & 5.630 \\
\hline NGC 5194 Enuc. 6 & 132939.32 & +470840.7 & $0.21 \pm 0.01$ & $0.50 \pm 0.10$ & $26.64 \pm 1.33$ & 12.321 \\
\hline NGC 5194 Enuc. 2 & 132944.10 & +471023.4 & $0.39 \pm 0.02$ & $1.40 \pm 0.28$ & $102.84 \pm 5.14$ & 6.834 \\
\hline NGC 5194 Enuc. 3 & 132945.13 & +470957.4 & $0.25 \pm 0.01$ & $0.67 \pm 0.13$ & $65.69 \pm 3.28$ & 7.048 \\
\hline NGC 5194 Enuc. 11 a & 132947.14 & +471341.3 & $0.10 \pm 0.01$ & $0.43 \pm 0.09$ & $14.18 \pm 0.71$ & 4.941 \\
\hline NGC 5194 Enuc. 11 b & 132947.58 & +471324.8 & $0.09 \pm 0.01$ & $0.13 \pm 0.03$ & $10.22 \pm 0.51$ & 4.340 \\
\hline NGC 5194 Enuc. 1 a & 132949.51 & +471240.3 & $0.38 \pm 0.02$ & $0.58 \pm 0.12$ & $84.19 \pm 4.21$ & 2.534 \\
\hline NGC 5194 Enuc. $11 \mathrm{~d}$ & 132949.58 & +47 1328.7 & $0.07 \pm 0.01$ & $0.12 \pm 0.02$ & $15.18 \pm 0.76$ & 4.078 \\
\hline NGC 5194 Enuc. $11 \mathrm{c}$ & 132949.67 & +471400.2 & $0.06 \pm 0.01$ & $0.10 \pm 0.02$ & $2.83 \pm 0.14$ & 5.221 \\
\hline NGC 5194c & 132950.02 & +471131.9 & $0.16 \pm 0.01$ & $0.32 \pm 0.06$ & $51.41 \pm 2.57$ & 1.804 \\
\hline NGC 5194 Enuc. $11 \mathrm{e}$ & 132950.64 & +471344.9 & $0.13 \pm 0.01$ & $0.26 \pm 0.05$ & $23.92 \pm 1.20$ & 4.633 \\
\hline NGC 5194b & 132951.64 & +471206.7 & $0.22 \pm 0.01$ & $0.60 \pm 0.12$ & $36.69 \pm 1.83$ & 0.977 \\
\hline NGC 5194 Enuc. 1 b & 132952.07 & +471243.6 & $0.27 \pm 0.02$ & $0.94 \pm 0.19$ & $76.18 \pm 3.81$ & 2.322 \\
\hline NGC $5194 \mathrm{e}$ & 132952.55 & +471152.6 & $0.45 \pm 0.02$ & $0.76 \pm 0.15$ & $30.02 \pm 1.50$ & 0.365 \\
\hline NGC $5194 d$ & 132952.73 & +471140.6 & $0.52 \pm 0.02$ & $1.86 \pm 0.37$ & $98.02 \pm 4.90$ & 0.092 \\
\hline NGC 5194 Enuc. $1 \mathrm{c}$ & 132953.13 & +47 1239.4 & $0.14 \pm 0.01$ & $0.67 \pm 0.13$ & $33.13 \pm 1.66$ & 2.323 \\
\hline NGC 5194 Enuc. 4 a & 132953.93 & +471404.9 & $0.08 \pm 0.01$ & $0.02 \pm 0.00$ & $10.79 \pm 0.54$ & 5.880 \\
\hline NGC 5194 Enuc. 10 a & 132955.35 & +471047.2 & $0.09 \pm 0.02$ & $0.18 \pm 0.04$ & $20.28 \pm 1.01$ & 2.336 \\
\hline NGC 5194 Enuc. 4 b & 132955.49 & +471401.6 & $0.19 \pm 0.01$ & $0.02 \pm 0.00$ & $34.81 \pm 1.74$ & 6.193 \\
\hline NGC 5194 Enuc. $4 \mathrm{c}$ & 132955.61 & +471350.2 & $0.08 \pm 0.01$ & $0.02 \pm 0.00$ & $13.88 \pm 0.69$ & 5.798 \\
\hline NGC 5194a & 132955.79 & +471145.1 & $0.34 \pm 0.02$ & $0.68 \pm 0.14$ & $108.62 \pm 5.43$ & 1.906 \\
\hline NGC 5194 Enuc. $10 \mathrm{~b}$ & 132956.52 & +471046.9 & $0.14 \pm 0.02$ & $0.39 \pm 0.08$ & $39.62 \pm 1.98$ & 2.723 \\
\hline NGC 5194 Enuc. 4 d & 132958.73 & +471409.4 & $0.07 \pm 0.01$ & $0.02 \pm 0.00$ & $23.43 \pm 1.17$ & 7.696 \\
\hline
\end{tabular}


Table 6

(Continued)

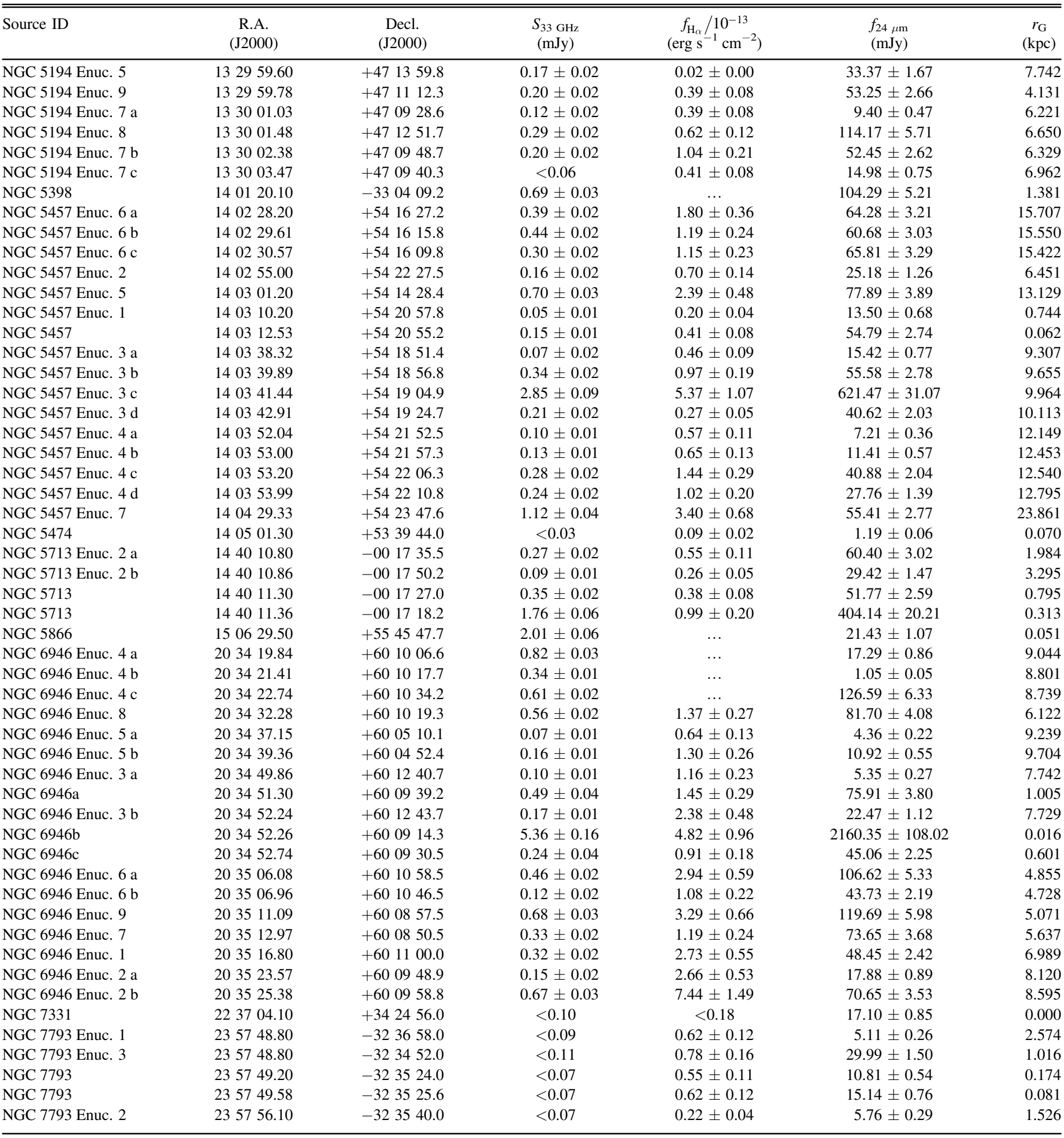

(This table is available in machine-readable form.)

IMSMOOTH. No astrometric alignment was necessary for the $24 \mu \mathrm{m}$ images, since the Spitzer astrometry was a near-perfect match to the VLA astrometry at 7 " resolution.

Figure 1 shows $\mathrm{H} \alpha$ and $24 \mu \mathrm{m}$ brightness contours overlaid on the $33 \mathrm{GHz}$ images. From a visual comparison, we find that at $7^{\prime \prime}(\sim 0.1-1 \mathrm{kpc})$ resolution, all but one strongly detected $(\geqslant 5 \sigma)$
$33 \mathrm{GHz}$ source has a $24 \mu \mathrm{m}$ counterpart with a nearly identical morphology. Such a tight morphological correlation is expected based on the well-known far-infrared (FIR)-radio correlation (de Jong et al. 1985; Helou et al. 1985). Studies of the resolved FIR-radio correlation (e.g., Hughes et al. 2006; Murphy et al. 2006; Tabatabaei et al. 2007a; Murphy et al. 2008) find that 
lower-frequency (synchrotron-dominated) radio emission is generally more spread out and diffuse than the corresponding dust emission associated with a single star-forming region as the result of $\mathrm{CR}$ electrons propagating significantly further than dust-heating photons. Since these $33 \mathrm{GHz}$ data are dominated by free-free emission rather than non-thermal synchrotron emission that traces propagating $\mathrm{CR}$ electrons, we expect this emission to remain more compact and closer to the skin of the $\mathrm{H}$ II regions where most of the warm dust emission is being powered.

At $2^{\prime \prime}(\approx 30-300 \mathrm{pc})$ resolution, we find only four $33 \mathrm{GHz}$ sources that are plausibly associated with star-forming regions and do not have $\mathrm{H} \alpha$ counterparts. Of these, two (NGC $4631 \mathrm{E}$ and NGC 4631 F; see Figure 1) are located in NGC 4631, an edge-on spiral galaxy where dust lanes are likely strongly affecting the observed spatial distribution of $\mathrm{H} \alpha$ emission. The first of the two remaining $33 \mathrm{GHz} / \mathrm{H} \alpha$ mismatches, NGC 3627 Enuc. 1 A, has a bright $33 \mathrm{GHz}$ peak that is morphologically distinct from any nearby $\mathrm{H} \alpha$ structure and is offset from the nearest $\mathrm{H} \alpha$ peak by $\approx 150 \mathrm{pc}$. However, the $24 \mu \mathrm{m}$ peak pixel is co-located with the $33 \mathrm{GHz}$ peak to better than $\approx 50$ pc. From this, we suspect that NGC 3627 Enuc. $1 \mathrm{~A}$ may be a highly extincted $\left(A_{\mathrm{H} \alpha} \gtrsim 5 \mathrm{mag}\right) \mathrm{H}$ II region. The final mismatch is NGC 5194 Enuc. $11 \mathrm{C}$, which is an unresolved radio peak located at the tip of a diffuse radio structure extending from the bright $\mathrm{H}$ II region NGC 5194 Enuc. $11 \mathrm{E}$. This source has neither an $\mathrm{H} \alpha$ nor a $24 \mu \mathrm{m}$ counterpart, which rules out dust as an explanation for the mismatch.

Our main result from this analysis is that $\approx 99 \%$ of the $33 \mathrm{GHz}$ sources in our sample have morphologically similar counterparts in both the $24 \mu \mathrm{m}$ (on scales of a few hundred pc) and $\mathrm{H} \alpha$ (on scales of $\sim 100 \mathrm{pc}$ ) images. The striking morphological similarities between the three tracers suggest that for each of these regions, the $\mathrm{H} \alpha, 24 \mu \mathrm{m}$, and $33 \mathrm{GHz}$ emission are powered by the same source, namely massive star formation. The $\mathrm{H} \alpha$ correspondence in particular suggests that the $33 \mathrm{GHz}$ emission is primarily powered by free-free emission. Another interesting implication of the $99 \%$ matching between $33 \mathrm{GHz}$ (and $24 \mu \mathrm{m}$ ) sources to $\mathrm{H} \alpha$ sources is that this places a relatively strong limit on the number of deeply embedded bright star-forming regions in these galaxies. Using $24 \mu \mathrm{m}$ and $\mathrm{H} \alpha$ observations, Prescott et al. (2007) report that $\approx 4 \%$ of their sources are "highly embedded" (i.e., $A_{\mathrm{H} \alpha} \gtrsim 3.3 \mathrm{mag}$ ) on $\approx 500 \mathrm{pc}$ scales for $\approx 1800$ star-forming regions. Using that same criterion, we find that $\approx 10 \%$ of our sources appear to be highly embedded (see Section 3.3). This is a slightly higher fraction than that reported by Prescott et al. (2007), which may be due to sampling regions at finer spatial scales (i.e., $\approx 100 \mathrm{pc}$ compared to $\approx 500 \mathrm{pc}$ ), or simply due to having much fewer sources in our analysis. If young clusters were buried in molecular clouds for a long period, we would expect to observe many $33 \mathrm{GHz}$ and $24 \mu \mathrm{m}$ sources without optical counterparts. Taking a typical $\mathrm{H}$ II region lifetime to be $\sim 5-10 \mathrm{Myr}$, our highly embedded fraction of $\approx 10 \%$ suggests that, on average, an $\mathrm{HII}$ region remains embedded for $\lesssim 1 \mathrm{Myr}$, consistent with multi-wavelength observations of young star-forming regions in a variety of extragalactic systems (e.g., Johnson et al. 2001; Whitmore et al. 2011).

\subsection{Radial Trends}

In Figure 3 we investigate if there are any trends in the ratio of the $33 \mathrm{GHz}$ flux to $\mathrm{H} \alpha$ line flux as a function of galactocentric radius. We distinguish nuclear from extranuclear

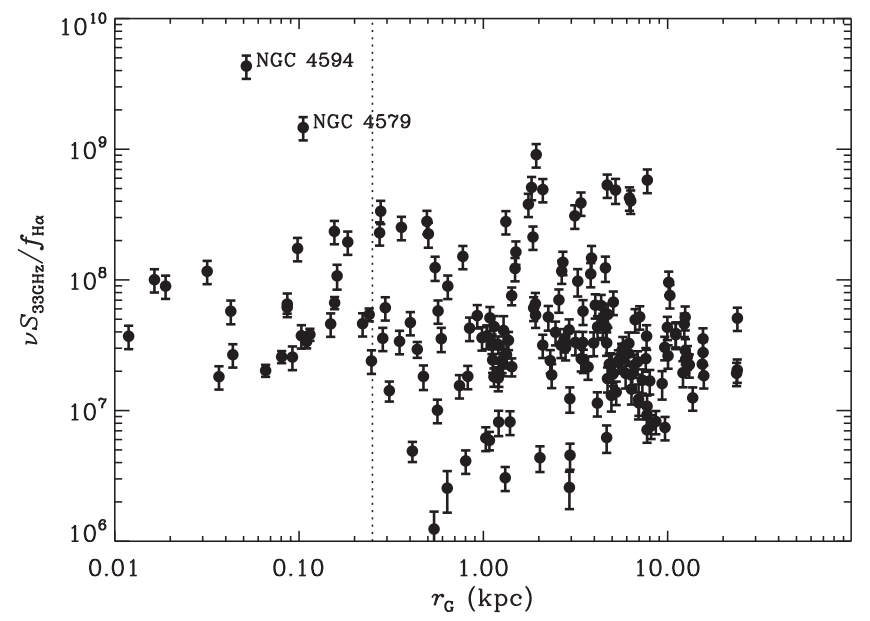

Figure 3. Ratio of $33 \mathrm{GHz}$ flux to $\mathrm{H} \alpha$ line flux plotted against galactocentric radius for all 162 sources having $\geqslant 3 \sigma$ detections at $33 \mathrm{GHz}$ and in $\mathrm{H} \alpha$. The vertical line at $r_{\mathrm{G}}=250 \mathrm{pc}$ indicates the radius used to conservatively distinguish nuclear and extranuclear regions, as some nuclear regions may be affected by AGNs. While no obvious trend is seen, the median ratio does appear to be statistically larger within a central diameter of $500 \mathrm{pc}$ for all galaxies than the outer disks by a factor of $1.82 \pm 0.39$. We identify those sources that are clear outliers, NGC 4594 and NGC 4579, which are both known to harbor AGNs that likely dominate the $33 \mathrm{GHz}$ continuum emission.

sources as having a galactocentric radius $r_{\mathrm{G}}<250 \mathrm{pc}$, since in some cases a fraction of the nuclear $33 \mathrm{GHz}$ emission may be powered by a central AGN (see Figure 3). For the 162 extranuclear sources detected at $>3 \sigma$ significance at both $33 \mathrm{GHz}$ and in $\mathrm{H} \alpha$, we calculate SFRs following the equations given in Murphy et al. (2011, 2012). As discussed in Section 3.1, given that these $33 \mathrm{GHz}$ data are able to resolve star-forming regions within each galaxy on $\approx 100 \mathrm{pc}$ scales, combined with the results of Murphy et al. (2012, 2015), we assume a $33 \mathrm{GHz}$ thermal fraction of $\approx 90 \%$ when calculating SFRs with Equation (11) in Murphy et al. (2011). Using the ratio of the optically-thin $33 \mathrm{GHz}$ to uncorrected $\mathrm{H} \alpha$ SFRs, we calculate a median extinction value on $30-300$ pc scales of $A_{\mathrm{H} \alpha} \approx 1.26 \pm 0.09 \mathrm{mag}$, similar to the value of $1.4 \mathrm{mag}$ reported by Prescott et al. (2007) when comparing $24 \mu \mathrm{m}$ and $\mathrm{H} \alpha$ photometry on $500 \mathrm{pc}$ scales for nearly 1800 star-forming regions within a sample of 38 nearby galaxies. The associated median absolute deviation is $0.87 \mathrm{mag}$. We believe that the rather large scatter here is driven by the corresponding large $(20 \%)$ calibration uncertainty associated with the difficulties in $\mathrm{H} \alpha$ narrowband imaging.

A strong trend in the $33 \mathrm{GHz}-$ to- $\mathrm{H} \alpha$ line flux ratio with galactocentric radius is not observed; however, the median ratio does appear to be statistically larger within the central $500 \mathrm{pc}$ diameter for all galaxies compared to the outer disks by a factor of $1.82 \pm 0.39$. Furthermore, a two-sided Kolmogorov-Smirnov (KS) test yields a probability of only $\approx 1.4 \%$ that both sets of ratios are drawn from the same distribution. With only the $33 \mathrm{GHz}$ and $\mathrm{H} \alpha$ data alone, it is unclear if this result is primarily due to a higher amount of non-thermal emission contributing to the $33 \mathrm{GHz}$ flux density or a larger amount of extinction attenuating the $\mathrm{H} \alpha$ emission within a galactocentric radius $r_{\mathrm{G}}<250 \mathrm{pc}$ for these galaxies. It is worth noting that there are studies in the literature showing that thermal fractions of circumnuclear star-forming regions are indeed lower relative to those in the outer disks of 
galaxies (e.g., Kennicutt et al. 1989; Murphy et al. 2011), which indicates that additional non-thermal emission likely plays a role, although there is significant scatter among sources (e.g., Murphy et al. 2012).

To attempt to break this degeneracy, we again plot the ratio of the $33 \mathrm{GHz}$ flux to $\mathrm{H} \alpha$ line flux as a function of galactocentric radius in the top panel of Figure 4 , as well as the ratio of the $33 \mathrm{GHz}$ to $24 \mu \mathrm{m}$ flux density as a function of galactocentric radius in the bottom panel, all at matched resolutions. Of the 179 discrete regions used for aperture photometry in the convolved maps, there are a total of 144 and 160 sources detected at $\geqslant 3 \sigma$ at $33 \mathrm{GHz}$ and $\mathrm{H} \alpha$ and $24 \mu \mathrm{m}$, respectively. In both panels we identify those sources with ratios that are clear outliers. These include NGC 4594 and NGC 4579, which are both known to harbor AGNs, NGC 6946 Enuc.4 B, which is a known AME detection (Murphy et al. 2010; Scaife et al. 2010; Hensley et al. 2015). The final source, NGC $4725 \mathrm{~B}$ has a spectrum that rises between 15 and $33 \mathrm{GHz}$ based on data to be published in a forthcoming paper. This may be indicative of another AME detection, but requires further investigation to see if this is indeed the case, or perhaps a background AGN peaking at $\gtrsim 33 \mathrm{GHz}$.

Similar to what is found in Figure 3 at higher resolution, the median ratio of $33 \mathrm{GHz}$ flux to $\mathrm{H} \alpha$ line flux does appear to be larger within a galactocentric radius $r_{\mathrm{G}}<250 \mathrm{pc}$ for all galaxies relative to the outer disk regions by a factor of $1.53 \pm 0.55$. A two-sided KS test yields a probability of $\approx 5 \%$ that both sets of ratios are drawn from the same distribution, which is less significant than the value measured at higher angular resolution above (i.e., $\approx 1.4 \%$ ). Assuming that the $33 \mathrm{GHz}$ and $24 \mu \mathrm{m}$ emission are both tracing current star formation unbiased by dust, any increase in this ratio among the nuclear versus the extranculear regions would suggest that the differences in the $33 \mathrm{GHz}$ flux and $\mathrm{H} \alpha$ line flux ratios are in fact due to an additional emission component powering the $33 \mathrm{GHz}$ emission (i.e., additional non-thermal emission). While we again find no obvious trend between the ratio of the $33 \mathrm{GHz}$ to $24 \mu \mathrm{m}$ flux densities versus galactocentric radius, the median ratio actually appears significantly smaller within a galactocentric radius $r_{\mathrm{G}}<250 \mathrm{pc}$ for all galaxies compared to the outer disks by a factor of $0.45 \pm 0.08$. A two-sided KS test in this case yields a probability of $\ll 1 \%$ that both sets of ratios are drawn from the same distribution. Consequently, there appears to be a larger amount of warm dust emission per unit star formation activity compared to $33 \mathrm{GHz}$ emission within the central $500 \mathrm{pc}$ diameter for the sample galaxies, consistent with far-infrared studies of nearby galaxies that find that dust tends to be warmer in the centers of galaxies (e.g., Tabatabaei et al. 2007b; Groves et al. 2012; Bendo et al. 2015).

Such a situation may arise if the circumnuclear regions of these galaxies have extended star formation history in which star formation that has taken place over a longer period of time, resulting in an accumulation of $\gtrsim 3 \mathrm{Myr}$ dust-heating stars in addition to any very old bulge stars that boost the $24 \mu \mathrm{m}$ flux density relative to the extranuclear regions. This is largely opposite to what we would expect if there was an additional component of non-thermal emission powering the $33 \mathrm{GHz}$ in the central regions of these galaxies, unless the excess dust-heating at $24 \mu \mathrm{m}$ far exceeds any additional non-thermal emission contribution at $33 \mathrm{GHz}$. So, while this result alone suggests that the larger ratio of $33 \mathrm{GHz}$ flux to $\mathrm{H} \alpha$ line flux found in the central regions of these galaxies may primarily arise from increased extinction, more detailed radio spectral fitting to obtain

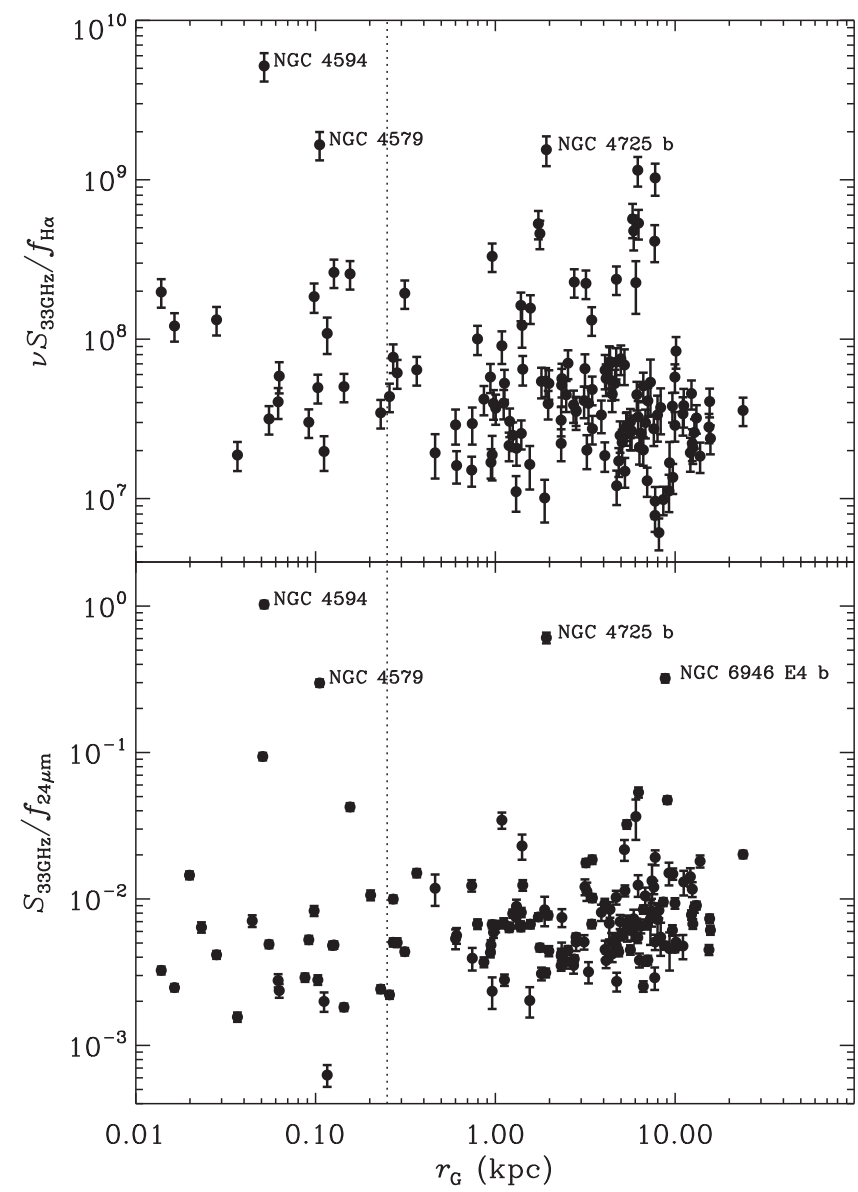

Figure 4. Top: ratio of $33 \mathrm{GHz}$ flux to $\mathrm{H} \alpha$ line flux plotted against galactocentric radius for all 144 sources having $\geqslant 3 \sigma$ detections at $33 \mathrm{GHz}$ and in $\mathrm{H} \alpha$ after convolving both data sets to $7^{\prime \prime}$ resolution to match the resolution of the $24 \mu \mathrm{m}$ Spitzer data. The vertical line at $r_{\mathrm{G}}=250 \mathrm{pc}$ (in both panels) indicates the radius used to conservatively distinguish nuclear and extranuclear regions, as some nuclear regions may be affected by AGNs. Similar to what is plotted in Figure 3 at higher resolution, no obvious trend is seen. However, the median ratio does appear to be larger within a galactocentric radius $r_{\mathrm{G}}<250 \mathrm{pc}$ for all galaxies than the outer disks by a factor of $1.53 \pm 0.55$. Bottom: ratio of $33 \mathrm{GHz}$ to $24 \mu \mathrm{m}$ flux density plotted against galactocentric radius for all 160 sources having $\geqslant 3 \sigma$ detections at $33 \mathrm{GHz}$ and $24 \mu \mathrm{m}$. Similar to the top panel, no obvious trend with galactocentric radius is seen. However, the median ratio does appear to be significantly smaller within a galactocentric radius $r_{\mathrm{G}}<250 \mathrm{pc}$ for all galaxies, compared to the outer disks, by a factor of $0.45 \pm 0.08$. In both panels we identify those sources that are clear outliers, NGC 4594 and NGC 4579, which are both known to harbor AGNs; NGC 6946 Enuc. 4 B, which is a known AME detection (Murphy et al. 2010; Scaife et al. 2010; Hensley et al. 2015); and NGC 4725 B, which may be a background AGN or another AME detection and warrants further investigation.

reliable thermal fractions is needed to help to confirm the dominant physical process driving the observed trend.

\section{Conclusions}

We have presented $33 \mathrm{GHz}$ interferometric imaging taken with the VLA for 112 fields (50 nuclei and 62 extranuclear H II regions) observed as part of the SFRS. These $\approx 2^{\prime \prime}$ resolution images are compared to archival $\mathrm{H} \alpha$ and $24 \mu \mathrm{m}$ imaging. Our conclusions can be summarized as follows.

1. A comparison with GBT single-dish $33 \mathrm{GHz}$ observations indicates that the interferometric VLA observations 
recover $78 \pm 4 \%$ of the total flux density over $25^{\prime \prime}$ regions $(\approx \mathrm{kpc}$ scales) among all fields on average, indicating that on the $\lesssim 300 \mathrm{pc}$ scales sampled by our VLA observations, missing emission from the lack of short spacings is not significant. On $\approx \mathrm{kpc}$ scales, the bulk of the emission being resolved out by our $33 \mathrm{GHz}$ interferometric observations is most likely diffuse nonthermal synchrotron emission associated with CR electrons as they propagate away from their birth sites in supernova remnants near $\mathrm{H}$ II regions. Consequently, on the $\approx 30-300$ pc scales sampled by our VLA observations the observed $33 \mathrm{GHz}$ emission is primarily powered by free-free emission from discrete $\mathrm{H}$ II regions, making it an excellent tracer of massive star formation.

2. A morphological comparison between the $33 \mathrm{GHz}$ radio, $\mathrm{H} \alpha$ nebular line, and $24 \mu \mathrm{m}$ warm dust emission shows remarkably tight similarities in their distributions, suggesting that each of these emission components are indeed powered by a common source (expected to be massive star-forming regions), and again suggests that the $33 \mathrm{GHz}$ emission is dominated by free-free emission.

3. Of the 225 discrete regions used for aperture photometry, 162 are detected at $>3 \sigma$ significance at both $33 \mathrm{GHz}$ and in $\mathrm{H} \alpha$ and are conservatively considered to be extranuclear and star-forming by having galactocentric radii $r_{\mathrm{G}} \geqslant 250 \mathrm{pc}$. By assuming a typical $33 \mathrm{GHz}$ thermal fraction of $90 \%$, we use this ratio of the optically-thin $33 \mathrm{GHz}$ to uncorrected $\mathrm{H} \alpha$ SFRs to calculate a median extinction value on $30-300 \mathrm{pc}$ scales of $A_{\mathrm{H} \alpha} \approx 1.26 \pm$ 0.09 mag with an associated median absolute deviation of $0.87 \mathrm{mag}$ among these star-forming regions.

4. We find that $\approx 99 \%$ of $33 \mathrm{GHz}$ sources in our sample have morphologically similar counterparts in both the $24 \mu \mathrm{m}$ (on scales of a few hundred parsecs) and $\mathrm{H} \alpha$ (on scales of $\sim 100 \mathrm{pc}$ ) images suggesting that each is powered by massive star formation. The $\mathrm{H} \alpha$ correspondence in particular suggests that the $33 \mathrm{GHz}$ emission is primarily powered by free-free emission. This result additionally puts a limit on the number of deeply embedded bright star-forming regions in these galaxies given that if young clusters were buried in molecular clouds for a long period, we would expect to observe many $33 \mathrm{GHz}$ and $24 \mu \mathrm{m}$ sources without optical counterparts. Our "highly embedded" (i.e., $A_{\mathrm{H} \alpha} \gtrsim 3.3 \mathrm{mag}$ ) fraction of $\approx 10 \%$ suggests that, on average, $\mathrm{H}$ II regions remain embedded for $\lesssim 1$ Myr.

5. We find that the median $33 \mathrm{GHz}$ flux to $\mathrm{H} \alpha$ line flux ratio is statistically larger within a galactocentric radius $r_{\mathrm{G}}<250 \mathrm{pc}$ for all galaxies relative to the outer disk regions by a factor of $1.82 \pm 0.39$. We additionally find that the median $33 \mathrm{GHz}$-to- $24 \mu \mathrm{m}$ ratio does appear to be statistically smaller in the central 500 pc diameter for all galaxies compared to the outer disk regions by a factor of $0.45 \pm 0.08$. The combination of these results suggests that the larger ratio of $33 \mathrm{GHz}$ flux to $\mathrm{H} \alpha$ line flux found in the central regions may arise primarily by increased extinction, rather than an excess of non-thermal radio emission. However, more detailed radio spectral fitting to obtain reliable thermal fractions is needed to help to confirm the dominant physical process driving this observed trend.
We would like to thank the anonymous referee for very useful comments that helped to improve the content and presentation of this paper. E.J.M. acknowledges the hospitality of the Aspen Center for Physics, which is supported by National Science Foundation grant No. PHY-1066293. The National Radio Astronomy Observatory is a facility of the National Science Foundation operated under cooperative agreement by Associated Universities, Inc. This research made use of APLpy, an opensource plotting package for Python hosted at http://aplpy. github.com.

\section{ORCID iDs}

E. J. Murphy (1) https://orcid.org/0000-0001-7089-7325

D. Dong (i) https://orcid.org/0000-0001-9584-2531

E. Momjian (10) https://orcid.org/0000-0003-3168-5922

S. Linden (10) https://orcid.org/0000-0002-1000-6081

D. S. Meier (1) https://orcid.org/0000-0001-9436-9471

E. Schinnerer (iD https://orcid.org/0000-0002-3933-7677

J. L. Turner (ib https://orcid.org/0000-0003-4625-2951

\section{References}

Aniano, G., Draine, B. T., Gordon, K. D., \& Sandstrom, K. 2011, PASP, 123,1218

Bendo, G. J., Baes, M., Bianchi, S., et al. 2015, MNRAS, 448, 135

Ciardullo, R., Feldmeier, J. J., Jacoby, G. H., et al. 2002, ApJ, 577, 31

Condon, J. J. 1992, ARA\&A, 30, 575

Condon, J. J., \& Yin, Q. F. 1990, ApJ, 357, 97

Conway, J. E., Cornwell, T. J., \& Wilkinson, P. N. 1990, MNRAS, 246, 490

Cornwell, T. J. 2008, ISTSP, 2, 793

Dale, D. A., Cohen, S. A., Johnson, L. C., et al. 2009, ApJ, 703, 517

Dale, D. A., Gil de Paz, A., Gordon, K. D., et al. 2007, ApJ, 655, 863

Dale, D. A., Giovanelli, R., Haynes, M. P., et al. 1997, AJ, 114, 455

de Jong, T., Klein, U., Wielebinski, R., \& Wunderlich, E. 1985, A\&A, 147, L6 de Vaucouleurs, G., de Vaucouleurs, A., Corwin, H. G., Jr., et al. 1991, Third

Reference Catalogue of Bright Galaxies. Vol. I, II, III (New York: Springer) Draine, B. T., \& Lazarian, A. 1998a, ApJL, 494, L19

Draine, B. T., \& Lazarian, A. 1998b, ApJ, 508, 157

Draine, B. T., \& Lazarian, A. 1999, ApJ, 512, 740

Engelbracht, C. W., Rieke, G. H., Gordon, K. D., et al. 2008, ApJ, 678, 804 Erickson, W. C. 1957, ApJ, 126, 480

Freedman, W. L., Madore, B. F., Gibson, B. K., et al. 2001, ApJ, 553, 47

Green, D. A. 2011, BASI, 39, 289

Groves, B., Krause, O., Sandstrom, K., et al. 2012, MNRAS, 426, 892

Helou, G., Soifer, B. T., \& Rowan-Robinson, M. 1985, ApJL, 298, L7

Hensley, B., Murphy, E., \& Staguhn, J. 2015, MNRAS, 449, 809

Hensley, B. S., Draine, B. T., \& Meisner, A. M. 2016, ApJ, 827, 45

Ho, L. C., Filippenko, A. V., \& Sargent, W. L. W. 1997, ApJS, 112, 315

Hughes, A., Wong, T., Ekers, R., et al. 2006, MNRAS, 370, 363

Jarrett, T. H., Chester, T., Cutri, R., Schneider, S. E., \& Huchra, J. P. 2003, AJ, 125,525

Johnson, K. E., Kobulnicky, H. A., Massey, P., \& Conti, P. S. 2001, ApJ, 559,864

Kennicutt, R. C., Calzetti, D., Aniano, G., et al. 2011, PASP, 123, 1347

Kennicutt, R. C., Jr., Armus, L., Bendo, G., et al. 2003, PASP, 115, 928

Kennicutt, R. C., Jr., Keel, W. C., \& Blaha, C. A. 1989, AJ, 97, 1022

Klein, U., \& Graeve, R. 1986, A\&A, 161, 155

Klein, U., Wielebinski, R., \& Morsi, H. W. 1988, A\&A, 190, 41

Kobulnicky, H. A., \& Johnson, K. E. 1999, ApJ, 527, 154

Koyama, K., Petre, R., Gotthelf, E. V., et al. 1995, Natur, 378, 255

Leroy, A. K., Bigiel, F., de Blok, W. J. G., et al. 2012, AJ, 144, 3

McMullin, J. P., Waters, B., Schiebel, D., Young, W., \& Golap, K. 2007, in ASP Conf. Ser. 376, Astronomical Data Analysis Software and Systems XVI, ed. R. A. Shaw, F. Hill, \& D. J. Bell (San Francisco, CA: ASP), 127

Mezger, P. G., \& Henderson, A. P. 1967, ApJ, 147, 471

Mohan, N., \& Rafferty, D. 2015, PyBDSM: Python Blob Detection and Source Measurement, Astrophysics Source Code Library, ascl:1502.007

Moustakas, J., Kennicutt, R. C., Jr., Tremonti, C. A., et al. 2010, ApJS, 190,233

Murphy, E. J., Braun, R., Helou, G., et al. 2006, ApJ, 638, 157

Murphy, E. J., Bremseth, J., Mason, B. S., et al. 2012, ApJ, 761, 97 
Murphy, E. J., Condon, J. J., Schinnerer, E., et al. 2011, ApJ, 737, 67

Murphy, E. J., Dong, D., Leroy, A. K., et al. 2015, ApJ, 813, 118

Murphy, E. J., Helou, G., Condon, J. J., et al. 2010, ApJL, 709, L108

Murphy, E. J., Helou, G., Kenney, J. D. P., Armus, L., \& Braun, R. 2008, ApJ, 678,828

Perley, R. A., \& Butler, B. J. 2013, ApJS, 204, 19

Planck Collaboration, Ade, P. A. R., Aghanim, N., et al. 2011, A\&A, 536, A20

Prescott, M. K. M., Kennicutt, R. C., Jr., Bendo, G. J., et al. 2007, ApJ, 668, 182

Rau, U., \& Cornwell, T. J. 2011, A\&A, 532, A71
Sault, R. J., \& Wieringa, M. H. 1994, A\&AS, 108, 585

Scaife, A. M. M., Nikolic, B., Green, D. A., et al. 2010, MNRAS, 406, L45

Tabatabaei, F. S., Beck, R., Krause, M., et al. 2007a, A\&A, 466, 509

Tabatabaei, F. S., Beck, R., Krügel, E., et al. 2007b, A\&A, 475, 133

Turner, J. L., \& Ho, P. T. P. 1983, ApJL, 268, L79

Turner, J. L., \& Ho, P. T. P. 1985, ApJL, 299, L77

Turner, J. L., \& Ho, P. T. P. 1994, ApJ, 421, 122

Turner, J. L., Ho, P. T. P., \& Beck, S. C. 1998, AJ, 116, 1212

Whitmore, B. C., Chandar, R., Kim, H., et al. 2011, ApJ, 729, 78 